\title{
Detection and characterization of the melting layer based on polarimetric radar scans
}

\author{
Daniel Wolfensberger, ${ }^{\mathrm{a}}$ Danny Scipion ${ }^{\mathrm{b}}$ and Alexis Berne ${ }^{\mathrm{a} \star}$ \\ ${ }^{a}$ Ecole Polytechnique Fédérale de Lausanne (EPFL), Lausanne, Switzerland \\ b Jicamarca Ionospheric Radio Observatory, Lima, Peru \\ ${ }^{*}$ Correspondence to: A. Berne, EPFL ENAC SSIE-GE, GR C2 564, Station 2, CH-1015 Lausanne, Switzerland. \\ E-mail: alexis.berne@epfl.ch
}

\begin{abstract}
Stratiform rain situations are generally associated with the presence of a melting layer characterized by a strong signature in polarimetric radar variables. This layer is an important feature as it indicates the transition from solid to liquid precipitation. The melting layer remains poorly characterized, particularly from a polarimetric radar point of view. In this work a new algorithm to automatically detect the melting layer on polarimetric RHI radar scans using gradients of reflectivity and copolar correlation is first proposed. The algorithm was applied to high-resolution X-band polarimetric radar data and validated by comparing the height of the detected layer with freezing-level heights obtained from radiosoundings and was shown to give both small errors and bias. The algorithm was then used on a large selection of precipitation events (more than 4000 RHI scans) from different seasons and climatic regions (South of France, Swiss Alps and plateau, and Iowa, USA) to characterize the geometric and polarimetric signatures of the melting layer. The melting layer is shown to have a very similar geometry on average, independent of the topography and climatic conditions. Variations in the thickness of the melting layer during and between precipitation events was shown to be strongly related to the presence of rimed particles, to the vertical velocity of hydrometeors and to the intensity of the bright band.
\end{abstract}

Key Words: melting layer; precipitation; polarimetric radar; stratiform

Received 16 February 2015; Revised 8 July 2015; Accepted 15 September 2015; Published online in Wiley Online Library

\section{Introduction}

The melting layer (ML) is an important feature of stratiform precipitation, associated with the melting of snowflakes/ice crystals below the freezing level that can be seen on precipitation radar scans as a thin, nearly horizontal layer with a high reflectivity factor, a feature known as the bright band (BB). The main cause of the $\mathrm{BB}$ effect is the fast increase in the dielectric constant of particles during the melting process, caused by the transition of the total water fraction within the ice-water mixture (Matrosov, 2008). The ML gives useful information about the vertical structure of precipitation since the base of the ML gives an indication of the vertical extent of liquid precipitation and the top of the ML is close to the altitude of the $0^{\circ} \mathrm{C}$ isotherm. The detection of the ML has been a long-standing topic of interest for radar meteorologists, mainly for quantitative precipitation estimation (QPE), because mixed-phase hydrometeors may contaminate rainfall estimates at longer distances (Giangrande et al., 2008). Moreover, the detection of the ML makes it possible to separate liquid from solid precipitation, which is critical information for hydrometeor classification algorithms. Finally, the ML is characterized by an important attenuation effect at $\mathrm{X}$-band and higher frequencies. Measurements at X-band by Bellon et al. (1997) showed that the attenuation effect of the ML could be $3-5$ times larger than the one caused by the rain below.
On modern radars equipped with dual polarimetry, the ML is characterized by a very distinct polarimetric signature (Figure 1). Besides the presence of large values of $Z_{\mathrm{H}}$ due to the $\mathrm{BB}$ effect, one notable characteristic of the ML on polarimetric scans is the presence of distinctly smaller values of the copolar cross-correlation coefficient $\rho_{\mathrm{hv}}$. Indeed, $\rho_{h v}$ depends on the homogeneity in shape of the hydrometeors and is significantly lower in the ML where phases are mixed, than in stratiform rain or in solid precipitation (e.g. Matrosov et al., 2007). One should keep in mind that low values of $\rho_{\text {hv }}$ can also be caused by non-meteorological echoes (e.g. insects, birds, aircraft). Additionally, the melting layer is also characterized by higher differential reflectivities $Z_{\mathrm{DR}}$ due to the transition between the solid phase where $Z_{\mathrm{DR}}$ is usually small (Doviak and Zrnić, 2006) to the liquid phase where it is higher. To summarize, the ML is characterized by the combination of a layer of small $\rho_{\mathrm{hv}}$ values, a transition from high to low $Z_{\mathrm{DR}}$ and the presence of high values in $Z_{\mathrm{H}}$ on polarimetric RHI scans.

Several operational algorithms for automatic detection of the ML on PPI scans have been proposed in the literature. Sánchez-Diezma et al. (2000) proposed an algorithm for BB detection from conventional operational radar scans based on the peak of reflectivity as well as the gradients of reflectivity between the $\mathrm{BB}$ and the liquid and solid phases. For polarimetric radars, Giangrande et al. (2008) proposed an algorithm for automatic ML detection in PPI scans, which searches for all range bins with 


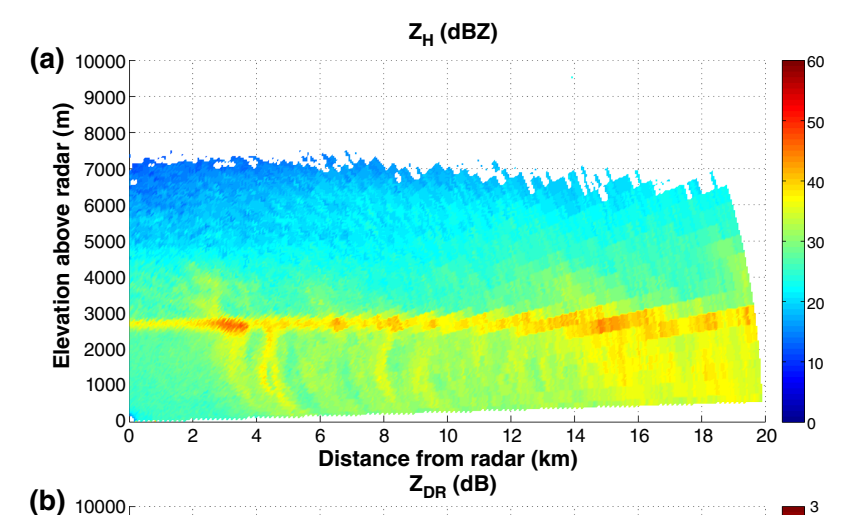

(b) 10000 $\mathrm{Z}_{\mathrm{DR}}(\mathrm{dB})$

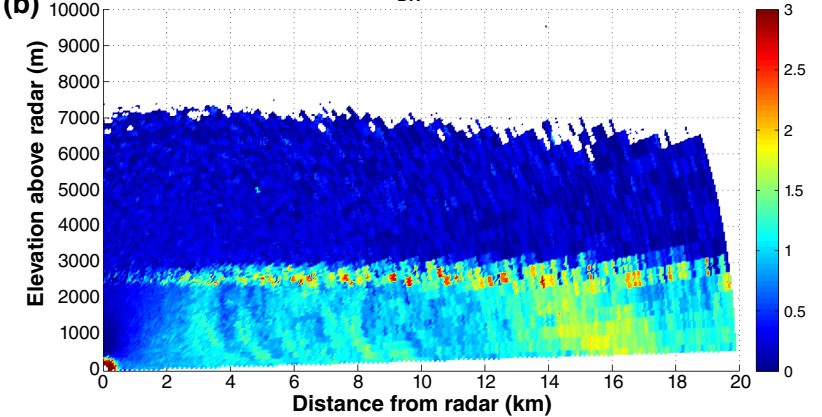

(c) 10000 .

$\rho_{\text {hv }}(-)$

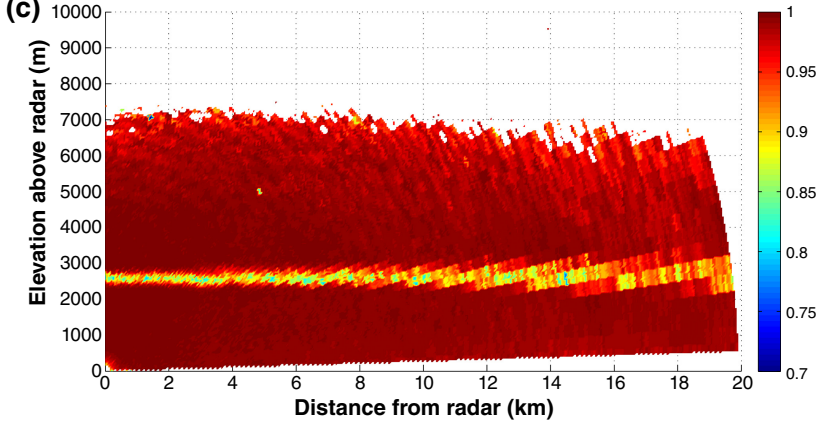

Figure 1. Example of the ML signature in (a) $Z_{\mathrm{H}}$, (b) $\rho_{\mathrm{hv}}$ and (c) ${ }^{\star} Z_{r m D R}$ in a typical stratiform rain situation collected in the south of France (29 September 2012 at 1224 UTC)

low $\rho_{h v}$ and classfies them as ML bins if the maxima of $Z_{H}$ and $Z_{D R}$ fall within a specified range. Matrosov et al. (2007) proposed a simpler approach, again in PPI where the boundaries of the ML are detected using only $\rho_{h v}$, a method also used by Kalogiros et al. (2013). The ML can also be detected by using Doppler velocities. White et al. (2002) propose a method using a wind profiler, which relies on the detection of the peak in reflectivity and zones where the gradient of reflectivity is negatively correlated with the vertical Doppler velocity. Few ML detection methods exist for RHI scans. Bandera et al. (1998) designed an algorithm that detects the ML based on the identification of strong vertical gradients in $Z_{H}$ and in the linear depolarization ratio $L_{D R}$, and assumes higher heterogeneity of radar variables within the ML.

Apart from its radar signature, the ML is also an important process as such. Some studies focused on the seasonal and geographical variability of the height of the ML. For example, Das et al. (1993) measured the variability of the ML height during 3 years in two different climatic regions of India. Although very common, the ML is still a relatively poorly known phenomenon and limited work has been done to date to study its scattering and geometric signatures. Fabry and Zawadzki (1995) analyzed vertical Doppler X-band radar and wind profiler data to quantitatively characterize the structure of the radar signature from melting precipitation. They suggested that the main cause of the BB were shape and density effects as well as the change in the refractive index of hydrometeors during melting. Zawadzki et al. (2005) developped a model of the melting snow and its radar reflectivity. A relationship between a large increase in velocity through the ML and a small reflectivity difference between the $\mathrm{BB}$ and the rain below was derived from the model and confirmed with vertically pointing radar observations. Durden et al. (1997) studied scans from a polarimetric airborne radar operated in the context of the TOGA COARE experiment over the Pacific ocean near New Guinea. The authors found some relation between the $\mathrm{BB}$ intensity and the distance between the maximum of reflectivity and the freezing level, which they explained by the latent cooling effect of melting. Additionally, they found a positive correlation between the BB intensity with both $\rho_{h v}$ and the vertical fall velocity within the ML.

These studies generally focused on one specific region and as such they might not be representative of the general characteristics of the ML. As an example, in tropical regions, the seasonal variations in thickness and altitude of the ML are expected to be weaker than at higher latitudes. Previous studies can also be complemented with the use of a hydrometeor classification scheme in order to gain a deeper understanding of the main factors that contribute to the ML variability.

Taking advantage of the strong polarimetric gradients at the ML boundaries, we propose a new algorithm for automatic ML detection on RHI polarimetric scans that is able to detect the height of boundaries of the ML all along the RHI. This algorithm is used to provide a more complete characterization and analysis of the structure and the polarimetric signature of the ML by using large datasets of polarimetric radar observations from different climatic regions (South of France, Western Switzerland, Swiss Alps and Iowa-USA). This study is completed by an in-depth analysis of the relationship between different characteristics of the ML.

This article is structured as follows: in section 2 the instruments and the datasets are described as well as all pre-processing operations transforming polar radar data into inputs to the ML detection algorithm. The algorithm is explained in detail in section 3 and is validated in section 4. Results of the characterization of the ML are given in section 5, which is divided into four parts focusing respectively on the attenuation effect, the vertical structure, the polarimetric signature and the geometry of the ML. These results are discussed in more details in section 6, which focuses on the relationship between ML descriptors, with an emphasis on the ML thickness. Finally, section 7 gives a summary of the main results and concludes this work.

\section{Data and processing}

\subsection{Instruments}

The radar measurements used in this work come from the EPFLLTE X-band polarimetric radar, called MXPol, as well as from a nearly identical radar system operated in the context of the NASA IFloodS* (Iowa Flood Studies) programme (Domaszczynski, 2012). Information about the characteristics of the polarimetric radar as well as its scanning strategy are given in Table 1.

For validation, data from the Swiss operational radiosoundings were used. These soundings are performed twice daily (0000 and 1200 UTC) from Payerne in western Switzerland and include measurements of temperature, pressure and relative humidity recorded every second. This corresponds to a vertical resolution of 5-10 m depending on the ascending velocity of the radiosonde.

\subsection{Datasets}

Since 2009, MXPol recorded a large amount of high-resolution polarimetric data during several measurement campaigns. In this work four datasets from different topographic and climatic regions are used; they are described in Table 2 and their locations are illustrated in Figure 2.

An interesting aspect is the high climatic diversity of the available datasets. The Ardèche region is characterized by a Mediterranean climate according to Köppen's classification (Peel

*http://gpm.nsstc.nasa.gov/ifloods/instruments.html; accessed 27 September 2015 . 
Table 1. MXPol features and scanning strategy.

\begin{tabular}{ll}
\hline Frequency & $9.4 \mathrm{GHz}$ \\
Range & $35 \mathrm{~km}$ \\
3dB beamwidth & $1.45^{\circ}$ \\
Rad. resolution & $75 \mathrm{~m}$ \\
Polarization & Simultaneous H-V \\
Scanning & PPIs at different elevations, \\
sequence & two RHIs and a vertical PPI \\
& (for $Z_{\mathrm{DR}}$ calibration) \\
Scanning & Interleaved pulse pair mode (PPI), \\
mode & Full Doppler Spectrum \\
& (RHI and vertical PPI) \\
\hline
\end{tabular}

et al., 2007). This climate is associated with warm summers and occasionally strong convective showers. Heavy precipitation events caused by the orographic updraught of wet air coming from the sea occur frequently in autumn. An overview of the climate of the Ardèche region and of the HyMeX (Hydrological Cycle in the Mediterranean experiment) program is given in Drobinski et al. (2014) and Ducrocq et al. (2014).

The region around Davos, in the Swiss Alps has a subarctic climate (Peel et al., 2007), with long and cold winters and mild summers. Precipitation mostly occurs in summer and early autumn due to orographic lifting and convection.

Iowa is part of the midwestern United States and is characterized by a humid continental climate (Peel et al., 2007) with marked seasonal variations. Summers are very warm and wet and are often associated with strong convection which can lead to the formation of supercells and tornados.

Finally, the last dataset was recorded in western Switzerland near the town of Payerne where the largest Swiss meteorological station is located. The radar set-up took place in the context of the PaRaDIso (PAyerne RADar and ISOtopes) programme which aims to study the segregation of isotopes in precipitation using combined sensors (disdrometers, radars, profilers). Compared with the Davos region, the Payerne region experiences a more oceanic climate (Peel et al., 2007) typical of western Europe, with limited seasonal temperature variability and milder winters. Precipitation occurs throughout the year with a maximum in late summer and autumn.

\subsection{Pre-processing of radar data}

\subsubsection{Radar variables and projections}

In the context of this work, five polarimetric radar variables are used: the reflectivity factor at horizontal polarization $Z_{\mathrm{H}}(\mathrm{dBZ})$, the copolar cross-correlation coefficient $\rho_{\mathrm{hv}}$, the differential reflectivity $Z_{\mathrm{DR}}(\mathrm{dB})$, the specific differential phase shift on propagation $K_{\mathrm{dp}}\left({ }^{\circ} \mathrm{km}^{-1}\right)$, and the radial velocity $V_{\mathrm{rad}}\left(\mathrm{m} \mathrm{s}^{-1}\right)$.

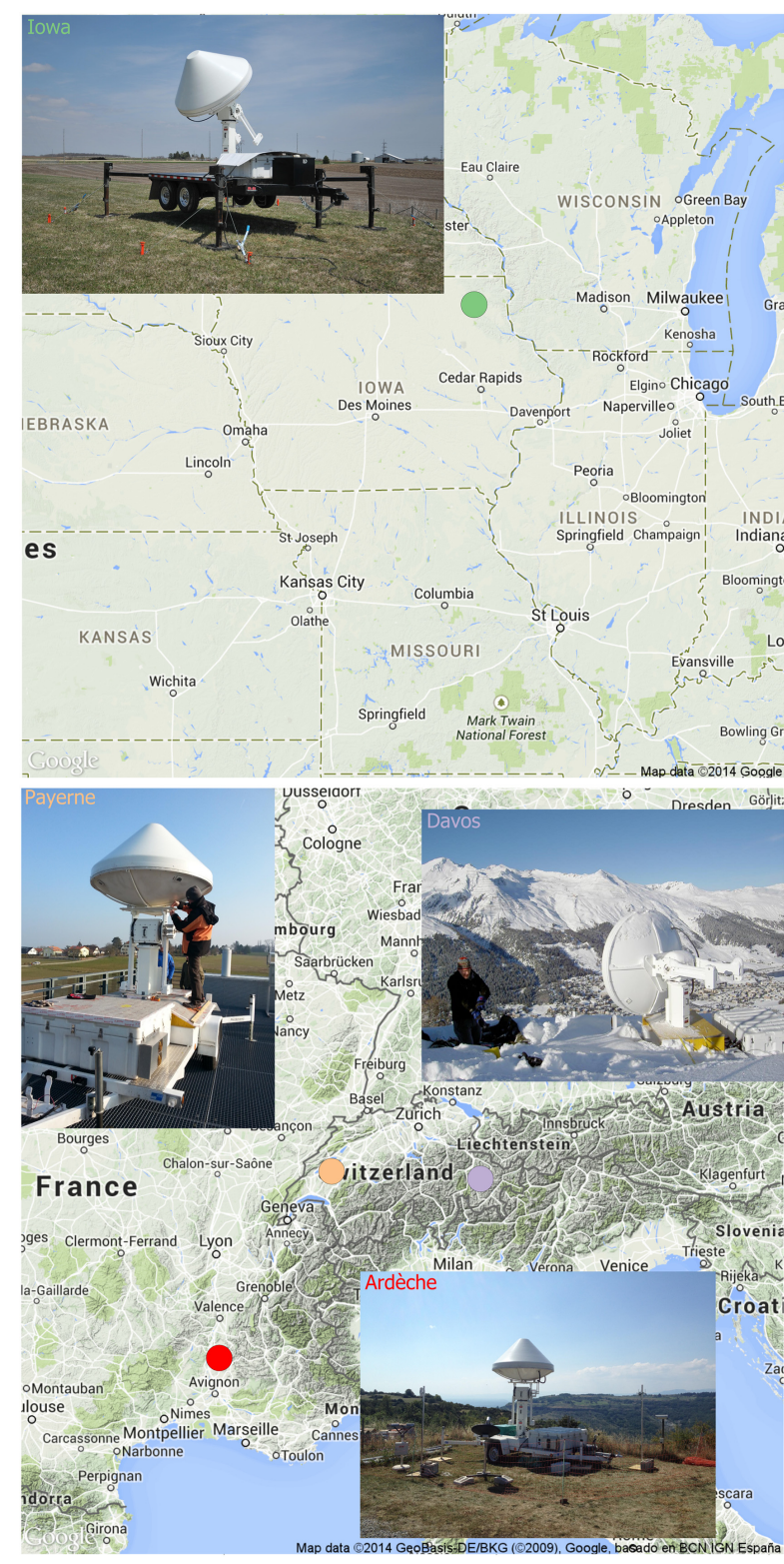

Figure 2. Locations and pictures of the four radar sites.

Only RHI scans are used, meaning that all variables are originally in polar range-elevation coordinates. Only measurements at a range shorter than $5 \mathrm{~km}$ are used in order to limit the effect of beam broadening and to consider only data with the highest signal-to-noise ratio. The choice of $5 \mathrm{~km}$ range can be justified by the fact that, at this distance, the diameter of the radar bin is approximately one third of the average ML thickness, which

Table 2. Description of the available RHI scans datasets.

\begin{tabular}{|c|c|c|c|c|c|c|}
\hline Site & Season & Context & $\begin{array}{l}\text { Altitude } \\
\text { (m amsl) }\end{array}$ & Coords. & $\begin{array}{c}\text { Topography } \\
\text { (altitude range) }\end{array}$ & Scans \\
\hline $\begin{array}{l}\text { Ardèche } \\
\text { (South of France) }\end{array}$ & $\begin{array}{l}\text { Autumn 2012, } \\
\text { Autumn } 2013\end{array}$ & $\begin{array}{l}\text { SOP1 and SOP2 }{ }^{a} \\
\text { of HyMEX }\end{array}$ & 605 & $\begin{array}{r}4.55^{\circ} \mathrm{E} \\
44.61^{\circ} \mathrm{N}\end{array}$ & $\begin{array}{l}\text { Small hills } \\
\text { and riverbeds } \\
(400-800 \mathrm{~m} \text { asl })\end{array}$ & $\begin{array}{l}1763 \mathrm{RHI} \\
\text { with ML }\end{array}$ \\
\hline $\begin{array}{l}\text { Davos } \\
\text { (Swiss Alps) }\end{array}$ & $\begin{array}{l}\text { Spring 2010- } \\
\text { summer } 2011\end{array}$ & $\begin{array}{l}\text { High altitude to } \\
\text { study mixed-phase } \\
\text { and solid precip. }\end{array}$ & 2133 & $\begin{array}{r}9.84^{\circ} \mathrm{E} \\
46.79^{\circ} \mathrm{N}\end{array}$ & $\begin{array}{l}\text { Complex terrain } \\
\text { across two valleys } \\
(1500-3000 \mathrm{~m} \text { asl })\end{array}$ & $\begin{array}{l}816 \mathrm{RHI} \\
\text { with ML }\end{array}$ \\
\hline $\begin{array}{l}\text { Iowa } \\
\text { (Midwestern USA) }\end{array}$ & Spring 2014 & $\begin{array}{l}\text { NASA IFloodS } \\
\text { campaign }\end{array}$ & 379 & $\begin{array}{l}91.86^{\circ} \mathrm{W} \\
43.18^{\circ} \mathrm{N}\end{array}$ & $\begin{array}{l}\text { Smooth flat terrain } \\
(200-400 \mathrm{~m} \text { asl })\end{array}$ & $\begin{array}{l}380 \mathrm{RHI} \\
\text { with ML }\end{array}$ \\
\hline $\begin{array}{l}\text { Payerne } \\
\text { (Swiss Plateau) }\end{array}$ & Spring 2014 & $\begin{array}{l}\text { PaRaDIso }^{c} \\
\text { campaign }\end{array}$ & 500 & $\begin{array}{r}6.94^{\circ} \mathrm{E} \\
46.81^{\circ} \mathrm{N}\end{array}$ & $\begin{array}{l}\text { Rolling grassland } \\
\text { between Jura and } \\
\text { Alps }(450-700 \mathrm{~m} \text { asl })\end{array}$ & $\begin{array}{l}507 \mathrm{RHI} \\
\text { with ML }\end{array}$ \\
\hline
\end{tabular}

${ }^{a}$ Special observing periods. ${ }^{b}$ Iowa Flood Studies, to assess the feasibility of flood forecasting using small satellite precipitation data. ${ }^{c}$ Payerne Radar and Isotopes, to study segregation of isotopes in precipitation. 
should still allow resolution of the ML with sufficient accuracy. The proposed approach should remain valid at longer ranges, but will suffer from beam broadening.

$K_{\mathrm{dp}}$ is estimated from the total differential phase shift $\Psi_{\mathrm{dp}}\left({ }^{\circ}\right)$ using a method based on Kalman filtering (Grazioli et al., 2014a; Schneebeli et al., 2014). This approach is designed to ensure the independence between $K_{\mathrm{dp}}$ estimates and other polarimetric variables, and to capture the fine-scale variations of $K_{\mathrm{dp}}$. Since this estimation of $K_{\mathrm{dp}}$ does not depend on $Z_{\mathrm{H}}$, it remains unaffected by the strong effect of the ML on $Z_{\mathrm{H}}$. All polarimetric variables are censored with a mask of signal-to-noise ratio of $8 \mathrm{~dB}$. Measurements at very low elevation angles $\left(0-2^{\circ}\right)$ are removed in order to avoid possible contamination by ground echoes. $Z_{\mathrm{DR}}$ measurements at high elevation angles $\left(45-90^{\circ}\right)$ are discarded, since they are strongly biased by the high angle of incidence of the radar beam (Ryzhkov et al., 2005).

The ML detection algorithm takes $Z_{\mathrm{H}}$ and $\rho_{\mathrm{hv}}$ projected onto a two-dimensional Cartesian grid as input. Projection from polar to Cartesian coordinates is done by simply assigning the value of the nearest radar bin to every cell of the Cartesian grid. If several radar bins fall into one Cartesian grid cell they are averaged (in linear values). In the context of this work, a small cell size of $25 \times 25 \mathrm{~m}^{2}$ is used to account for the higher density of radar bins at short range. This cell size has been chosen as a compromise between calculation time and accuracy of the Polar to Cartesian projection. Tests showed that changing the interpolation grid size between 25 and $75 \mathrm{~m}$ does not bias the results presented in section 5 .

\subsubsection{Attenuation correction}

In the liquid phase, the attenuation correction for $Z_{\mathrm{H}}$ and $Z_{\mathrm{DR}}$ is directly calculated in polar data according to Testud et al. (2000), using the relations linking $K_{\mathrm{dp}}, Z_{\mathrm{H}}$, the specific horizontal attenuation $\alpha_{\mathrm{H}}\left(\mathrm{dB} \mathrm{km}^{-1}\right)$, and the specific differential attenuation $\alpha_{\mathrm{DR}}\left(\mathrm{dB} \mathrm{km}^{-1}\right)$. The power laws linking the variables were obtained using simulated realistic drop-size distribution fields (Schleiss et al., 2012). Since the attenuation properties in the melting layer are not known precisely, the attenuation correction is calculated only in the liquid phase and the correction is simply propagated further above, using the ML detection algorithm (section 3) as reference to detect the base of the ML. Neglecting the attenuation in the solid phase should be acceptable since it is usually much smaller than in the liquid phase (Doviak and Zrnić, 2006). The situation is quite different in the ML where significant attenuation may occur (Bellon et al., 1997). More information about the ML attenuation effect is provided in section 5.1.

\subsubsection{Hydrometeor classification}

In order to gain a better understanding of the ML signature, a hydrometeor classification is performed in the solid phase above the detected ML using the classification algorithm of Grazioli et al. (2014b). This algorithm takes $Z_{\mathrm{H}}, Z_{\mathrm{DR}}, \rho_{\mathrm{hv}}$ and $K_{\mathrm{dp}}$ as well as an estimation of the freezing-level height as input and classifies every pixel into one of seven classes, light rain (LR), rain (R), heavy rain (HR), melting snow (MS), ice crystals/small aggregates $(\mathrm{CR})$, aggregates (AG) and rimed particles (RI). In the context of this work, the height of the top of the detected ML was used as an estimation of the freezing-level height.

A flowchart summarizing all the pre-processing steps is shown in Figure 3.

\section{Automatic detection of the ML}

\subsection{Description of the algorithm}

Instead of simply adapting an algorithm designed for PPI scans, a new algorithm was designed that works directly in RHI scans by taking advantage of the fact that vertical gradients in $\rho_{\mathrm{hv}}$ and $Z_{\mathrm{H}}$ are usually large and well defined. $Z_{\mathrm{DR}}(\mathrm{dB})$ is not used in the

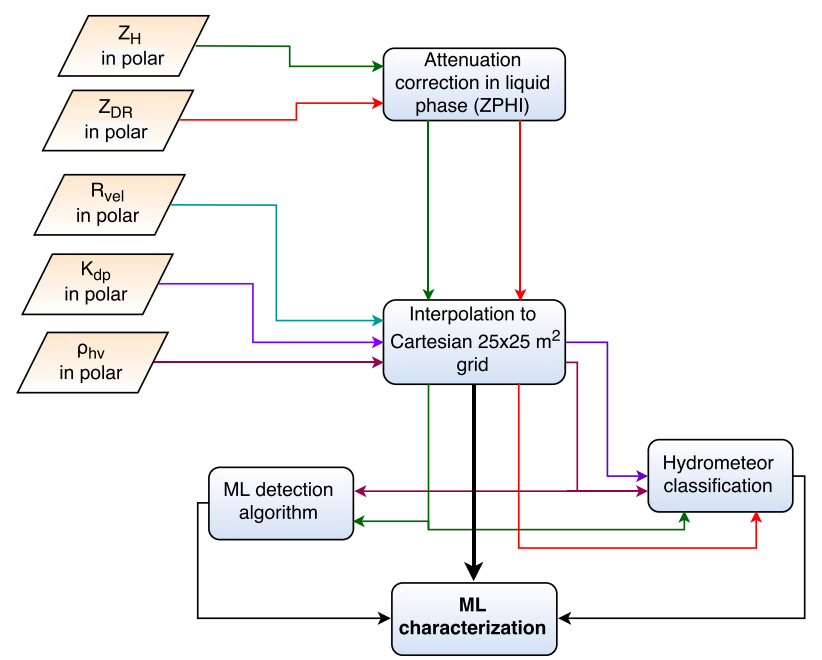

Figure 3. Flowchart of the preprocessing steps.

current algorithm because it is ill-defined at high elevation angles and because no improvement was observed when adding $Z_{\mathrm{DR}}$ as a third input variable.

The main advantage of this algorithm is that it estimates the ML boundaries all along the vertical profile at a high resolution. As the main motivation of the present work is the characterization of the ML, we only consider radar data at short range ( $5 \mathrm{~km} \mathrm{max}$ ), in order to have reliable high-resolution observations not affected by beam broadening.

The algorithm is divided into two parts. First, an initial estimation of the ML is obtained by using both $Z_{\mathrm{H}}$ and $\rho_{\mathrm{hv}}$ and by assuming that the ML is a more or less horizontal structure. This first part of the algorithm is similar to Bandera et al. (1998), the main difference being the use of $\rho_{\mathrm{hv}}$ instead of $L_{\mathrm{DR}}$.

This initial estimation which corresponds mostly to the layer of low $\rho_{\mathrm{hv}}$ can sometimes underestimate the extent of the ML. Indeed, $Z_{\mathrm{H}}$ starts to increase when the ice crystals start to melt, i.e. when they are still large but contain a significant amount of liquid water; however $\rho_{\mathrm{hv}}$ decreases significantly only when the mixture between ice crystals and drops is already quite heterogeneous. This happens at a lower altitude, when sufficient melting has already occurred. Generally the distance between the maximum in $Z_{\mathrm{H}}$ and the minimum in $\rho_{\mathrm{hv}}$ increases with the concentration of hydrometeors (e.g. Giangrande et al., 2008). In the case of intense precipitation, the top of the ML may thus be above the layer of lower values of $\rho_{\mathrm{hv}}$. The second part of the algorithm aims to alleviate this effect: the top of the ML is estimated using the same procedure but with gradients in $Z_{\mathrm{H}}$ only.

All steps of the algorithm are explained in detail below. Justification for the chosen values for the parameters of the algorithm will be given in section 3.3.

\section{Part 1: initial estimation}

1. $Z_{\mathrm{H}}$ and $\rho_{\mathrm{hv}}$ are normalized: $[10,60] \mathrm{dBZ} \rightarrow[0,1]$ for $Z_{\mathrm{H}}$ and $[0.65,1] \rightarrow[0,1]$ for $\rho_{\mathrm{hv}}$, in order to give a similar weight to both variables. These boundaries correspond to the range of values expected in precipitation. Experiments showed that changing this range slightly, e.g. using $[0,60]$ instead of $[10,60]$, does not change the output of the algorithm.

2. The normalized variables are then combined into a single image:

$$
\mathrm{IM}_{\mathrm{comb}}=Z_{\mathrm{H}} \cdot\left(1-\rho_{\mathrm{hv}}\right)
$$

Note that, since the ML is characterized by high values of reflectivity and small values of $\rho_{\mathrm{hv}}$, the complement of $\rho_{\mathrm{hv}}$ is used in the product. 
3. The vertical gradient of the image is computed using a classical vertical Sobel filter: ${ }^{\dagger}$

$$
h_{\text {Sobel }}=\left[\begin{array}{rrr}
-1 & -2 & -1 \\
0 & 0 & 0 \\
1 & 2 & 1
\end{array}\right] \text {. }
$$

To decrease the noise, the image is filtered with a moving average of length $L_{\text {filt,grad }}$ (in practice a length of $75 \mathrm{~m}$ is used, which corresponds to $75 \times 75 \mathrm{~m}^{2}$, i.e. a window of $3 \times 3$ pixels).

4. The gradient image is thresholded. All pixels with absolute value larger than $T_{\text {grad,min }}$ (set to 0.02 ) are kept, whereas all others are set to 0 . This step is done in order to detect only gradient extremes that are strong enough to correspond to a potential ML edge.

5. The image is scanned column by column (i.e. a vertical profile). The minimum and maximum of the vertical gradient are detected for each column. The lower edge of the ML is associated with the maximum and the upper edge with the minium.

6. The median height of the upper boundary of the ML $\left(\mathrm{Med}_{M L, \text { top }}\right)$ and the median height of the lower boundary of the ML $\left(\operatorname{Med}_{M L, \text { bot }}\right)$ are computed at the end of this step.

7. Step 5 is run again, but this time after discarding the gradient image above $\left(1+f_{\mathrm{ML} \text {,height }}\right) \cdot \operatorname{Med}_{\mathrm{ML} \text {,top }}$ and below $\left(1-f_{\text {ML,height }}\right) \cdot$ Med $_{\text {ML,bot, assuming the ML is a }}$ relatively flat structure. This helps to remove the possible contamination by ground echoes or small embedded cells of intense rainfall. The chosen value for $f_{\mathrm{ML} \text {,height }}$ is 0.3 .

\section{Part 2: correction of the ML top}

8. The vertical gradient is calculated as in step 3, but on the normalized $Z_{\mathrm{H}}$ image only.

9. For every vertical column, the gradient image is cut below the top of the ML calculated in Part 1 (Figure 4, point 2) and above the first local maximum in the gradient in the solid phase (Figure 4, point 4).

10. Using this new gradient image, the top of the ML is detected again as in steps $5-7$ of Part 1 .

11. (Optional) Small gaps in the ML are filled if their size is smaller than $250 \mathrm{~m}$ (section 3.3). Interpolation is done separately on the lower and upper boundaries of the ML using shape-preserving piecewise Hermite interpolation polynomials ('pchip' in Matlab).

An illustration of the behaviour of the gradient of $Z_{\mathrm{H}}, \rho_{\mathrm{hv}}$ and $Z_{\mathrm{H}}\left(1-\rho_{\mathrm{hv}}\right)$ along a vertical profile is given in Figure 4 . Point 1 corresponds to a positive peak in the gradient of $Z_{\mathrm{H}} \cdot\left(1-\rho_{\mathrm{hv}}\right)$ and is associated with the bottom of the ML. Point 2 corresponds to a negative peak in the gradient of $Z_{\mathrm{H}} \cdot\left(1-\rho_{\mathrm{hv}}\right)$ and a positive peak in the $\rho_{\mathrm{hv}}$ gradient. It marks the upper edge of the layer of low $\rho_{\mathrm{hv}}$ values. Points 1 and 2 are detected at the end of the first part of the algorithm (step 7). Point 2 is generally lower in altitude than the freezing level due to concentration effects. Point 3 corresponds to a negative peak in the $Z_{\mathrm{H}}$ gradient which marks the upper bound of the $\mathrm{BB}$ and is closer to the real height of the freezing level. This point is considered as the top of the $\mathrm{ML}$ and is detected at the end of the second part of the algorithm (step 10).

The gradient of $Z_{\mathrm{H}}$ is generally low in the solid phase and oscillates around zero, while it is highly variable in the liquid phase. The gradient of $\rho_{\mathrm{hv}}$ is mostly 0 in the liquid and solid phases. Points 4 and 5 illustrate why the gradient is cut above the first local maximum in step 9 of the algorithm; it can happen

\footnotetext{
${ }^{\dagger}$ Compared with a simple 1D finite difference, the Sobel operator is les sensitive to isolated high-intensity point variations thanks to the local horizonta averaging over sets of three pixels (Wenshuo et al., 2010).
}

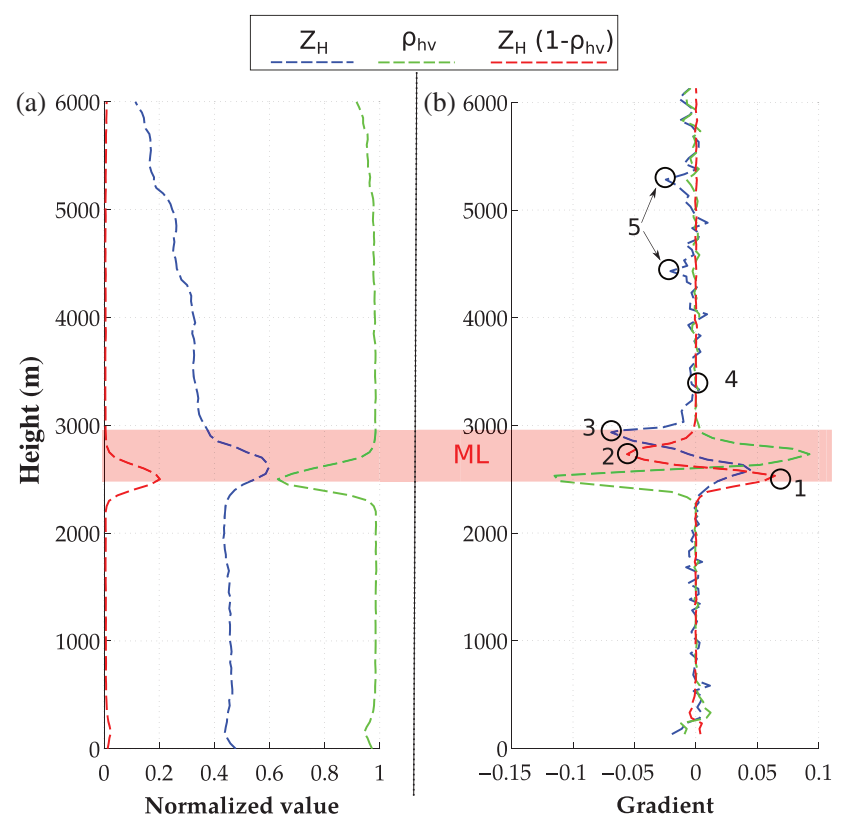

Figure 4. (a) Normalized values and (b) gradients of $Z_{\mathrm{H}}$ and $\rho_{r m h v}$ and the combined image at the boundaries of the ML on a RHI scan recorded at Ardèche (29 September 2012).

that due to some layer of higher $Z_{\mathrm{H}}$ in the solid phase (in case of riming for example) the gradient decreases again to reach a secondary minima as in the points 5 . Clearly these values do not correspond to the ML top and may in some very rare cases be even stronger than the $Z_{\mathrm{H}}$ gradient signature of the ML top. To alleviate this effect, the search for the minimum is stopped as soon as a first local maximum (point 4 ) is encountered.

Figure 5 presents a flow chart of the proposed algorithm. In summary, $Z_{\mathrm{H}}$ and $\rho_{\mathrm{hv}}$ are first normalized and combined. The vertical gradient of the combined image is then calculated and thresholded. In a first approximation, the upper and lower boundaries of the ML are identified by the minimum and the maximum of the vertical gradient. This first approximation is then refined by detecting again the upper boundary based on the gradient of $Z_{\mathrm{H}}$ only.

\subsection{Outputs}

Two examples of ML detection during stratiform situations of different intensities are shown in Figure 6. The bottom of the detected ML matches well the sharp transition to smaller values of $\rho_{\mathrm{hv}}$ inside the ML and the top of the ML corresponds well with the top of the $\mathrm{BB}$. The second case shows that the algorithm also has a good sensitivity since even a weak ML can be detected. Small-scale fluctuations of the ML are also accurately detected.

Thanks to the algorithm, it is possible to estimate the distribution of polarimetric variables in the liquid phase (below the ML) and the solid phase (above). The distribution of $\rho_{\mathrm{hv}}$ over all datasets (Figure 7) shows that $\rho_{\mathrm{hv}}$ within the detected ML is much lower than within the liquid and solid phases, which indicates that the detected ML corresponds to a region of much larger hydrometeor variability, consistent with the presence of melting. A more quantitative analysis and evaluation of the algorithm is provided in section 5 .

\subsection{Algorithm parameters}

The algorithm relies on four independent parameters which are given in Table 3. The recommended values were first chosen empirically and then verified based on sensitivity and statistical analysis in order to assess the potential associated uncertainty.

The first parameter of importance is $T_{\text {grad,min }}$, the threshold on gradient magnitude. The value of 0.02 was chosen by visual 


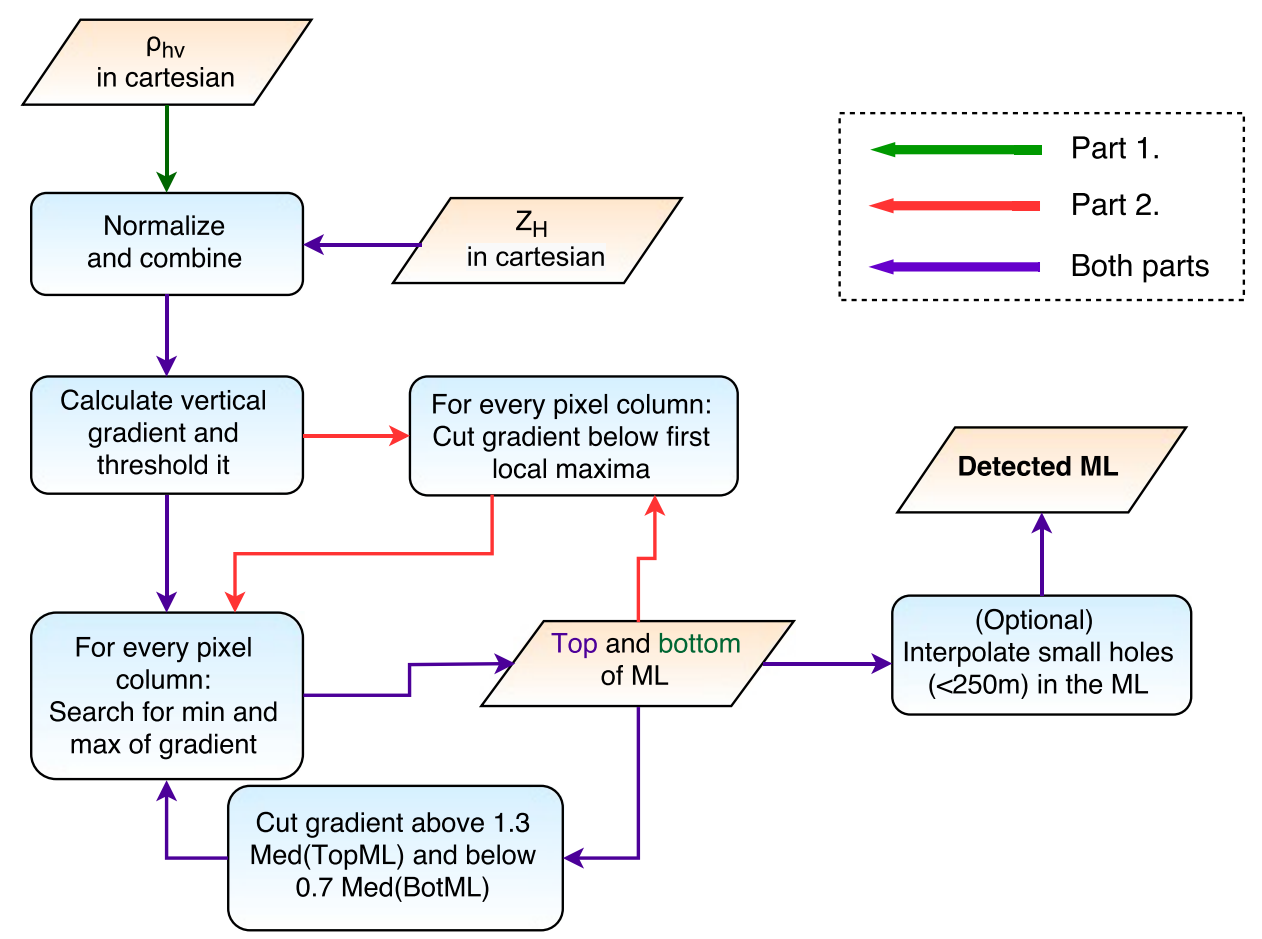

Figure 5. Flow diagram of the ML detection algorithm.
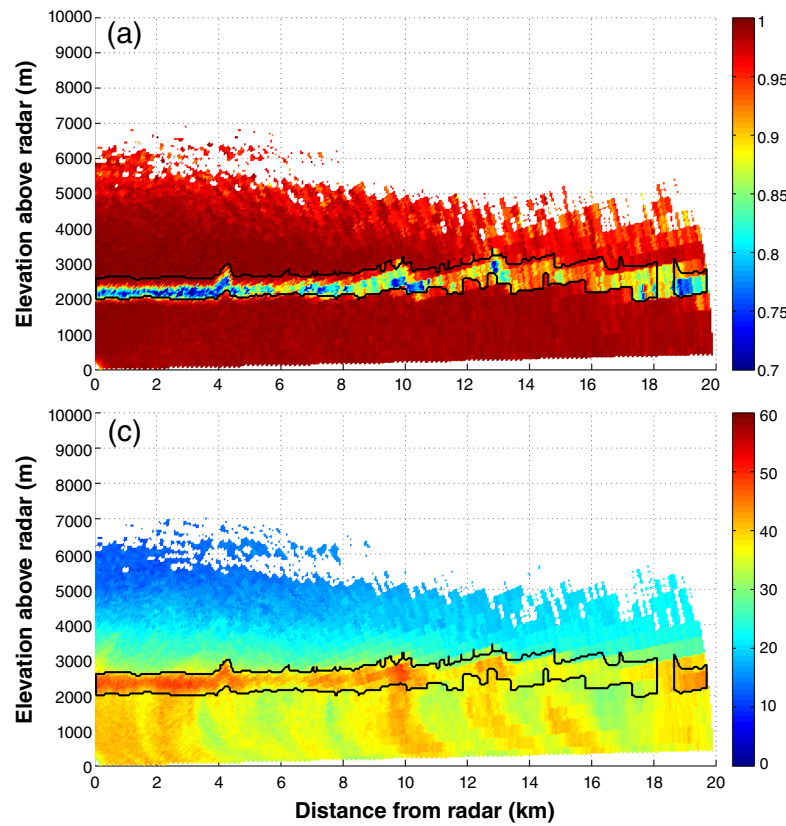

Figure 6. Two examples of ML detection overlaid on (a,b) $\rho_{\mathrm{hv}}$ and $(\mathrm{c}, \mathrm{d}) Z_{\mathrm{H}}$.

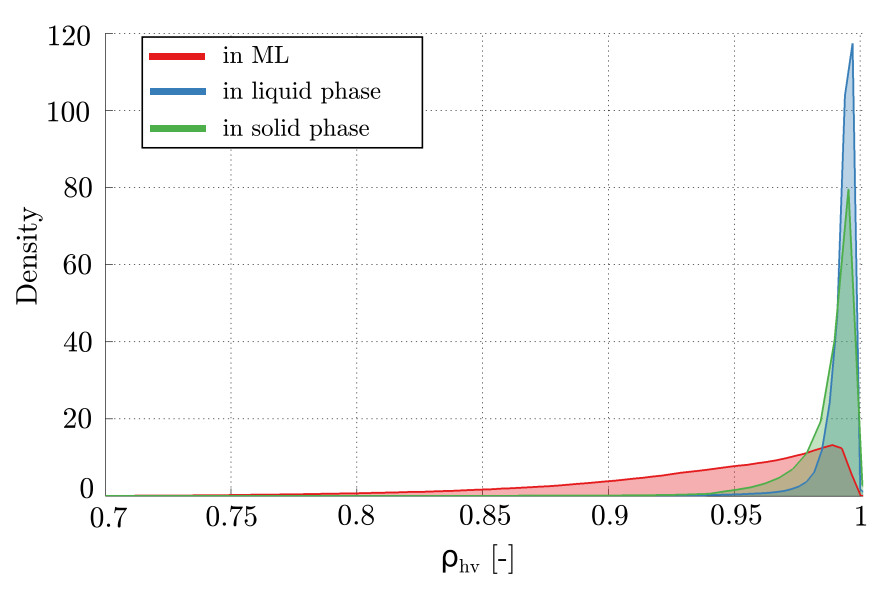

Figure 7. Distributions of $\rho_{\mathrm{hv}}$ in the liquid and solid phases and in the identified ML.
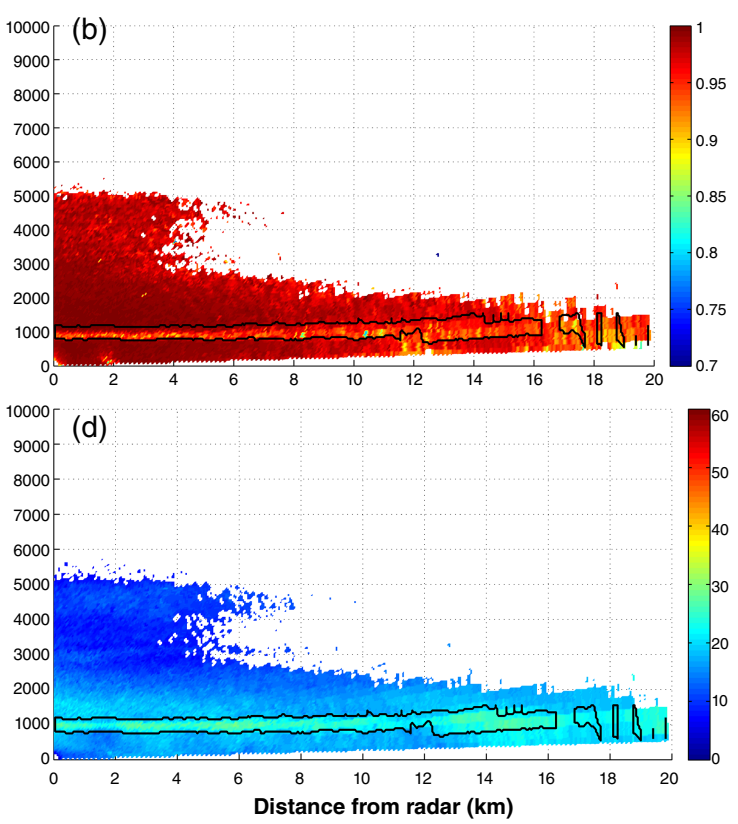

inspection as it was found that values of this magnitude are very rarely observed in situations without a ML. It is meant to avoid considering edges of too low intensity. It was observed that this constraint does not negatively affect the detection even for relatively weak ML situations. Increasing the threshold will lead to fewer pixels being detected and to gaps in the detected ML, but reduces the risk of erroneous detection. However, the output of the algorithm is not very sensitive to small variations of this parameter because gradients caused by the ML are many orders of magnitude larger than gradients below or above the ML. To verify this, the algorithm was run on all RHI scans (from all datasets) using values of $T_{\text {grad,min }}$ ranging from 0.005 to 0.035 . For every threshold value, an agreement score with the reference ( $T_{\text {grad,min }}$ $=0.02$ ) was calculated

$$
\operatorname{Score}(k)=2 \frac{\sum_{i=0}^{N} \sum_{j=0}^{M}\left(M L_{T_{\text {grad,min }=k}}^{i, j} \cap M L_{T_{\text {grad,min }=0.02}}^{i, j}\right)}{\sum_{i=0}^{N} \sum_{j=0}^{M}\left(M L_{T_{\text {grad,min }=k}}^{i, j}+M L_{T_{\text {grad,min }=0.02}}^{i, j}\right)},
$$


Table 3. Algorithm parameters and recommended values.

\begin{tabular}{llcc}
\hline Parameter & Meaning & Value & Unit \\
\hline$L_{\text {filt,grad }}$ & $\begin{array}{l}\text { Size of moving average filter } \\
\text { for gradient smoothing } \\
\text { Threshold on gradient } \\
T_{\text {grad,min }}\end{array}$ & 75 & $\mathrm{~m}$ \\
$f_{\mathrm{ML}, \text { height }}$ & $\begin{array}{l}\text { Magnitude } \\
\text { Maximum allowable relative } \\
\text { fluctuation of ML top and } \\
\text { bottom } \\
L_{\text {gaps,max }}\end{array}$ & 0.02 & $\mathrm{~m}^{-1}$ \\
\hline
\end{tabular}

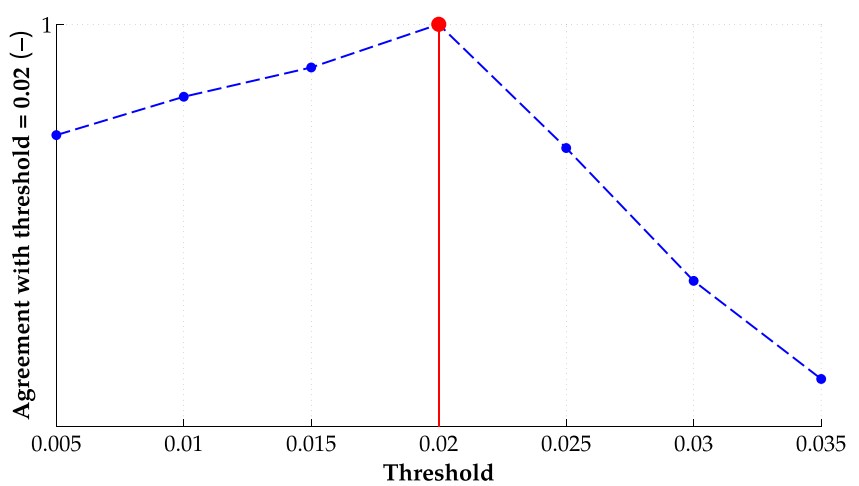

Figure 8. Sensitivity of the algorithm output to variations of $T_{\text {grad, min }}$ (gradient threshold) with respect to the reference threshold $(0.02)$, shown by the red marker.

where $N$ and $M$ are the dimensions of the Cartesian radar scan grid.

In other words, the agreement score is twice the number of pixels that are classified as the ML for both gradient values divided by the sum of the number of ML pixels detected for every single threshold value. A value of 1 means a perfect agreement and 0 a total disagreement (the two MLs do not overlap). Figure 8 shows the agreement for every chosen threshold value. Generally the agreement is quite good (more than $90 \%$ ), which shows that the detected MLs do not differ much.

The second parameter is the constraint on the relative height of the bottom and the top of the ML. In the algorithm, it is assumed that the bottom of the ML does not fluctuate below $\left(1-f_{\mathrm{ML} \text {,height }}\right) \cdot \operatorname{Med}_{\mathrm{ML}, \text { bottom }}$ and the top of the ML not above $\left(1+f_{\mathrm{ML}, \text { height }}\right) \cdot \operatorname{Med}_{\mathrm{ML}, \text { top }}$. The chosen value of $f_{\mathrm{ML} \text {,height }}=0.3$ was first determined by visual inspection. To test its relevance, the top and bottom relative heights of the ML were computed on all available RHI scans for a maximum distance from the radar going up to $35 \mathrm{~km}$ (the radar maximum range). The relative heights are defined by:

$$
M L_{\text {top,rel }}=\frac{M L_{\text {top }}}{M e d_{\mathrm{ML}, \text { top }}} \text { and } M L_{\text {bot,rel }}=\frac{M L_{\text {bot }}}{M e d_{\mathrm{ML}, \text { bot }}} .
$$

The distributions of the relative heights are shown in Figure 9. The cutting limits of 0.7 and 1.3 are displayed as red lines. The histograms are symmetrical and do not seem to be truncated near the cutting limits. The fluctuations stay generally well below the red limits, even though at $35 \mathrm{~km}$ range the beam broadening effect is quite important. The recommended value of 0.3 can thus be considered as appropriate and robust.

The third parameter is the maximum size of gaps that can be interpolated, $L_{\text {gaps,max }}$. It often happens that, on the whole scan, a couple of pixels are not detected which leads to small holes in the detected ML. In those cases, interpolating small gaps could be considered as a valid option. In order to set a limit to the maximum size of gaps that should be interpolated, the distribution of gap sizes within the ML was computed. It can be seen on Figure 10, that the vast majority of gaps are rather small
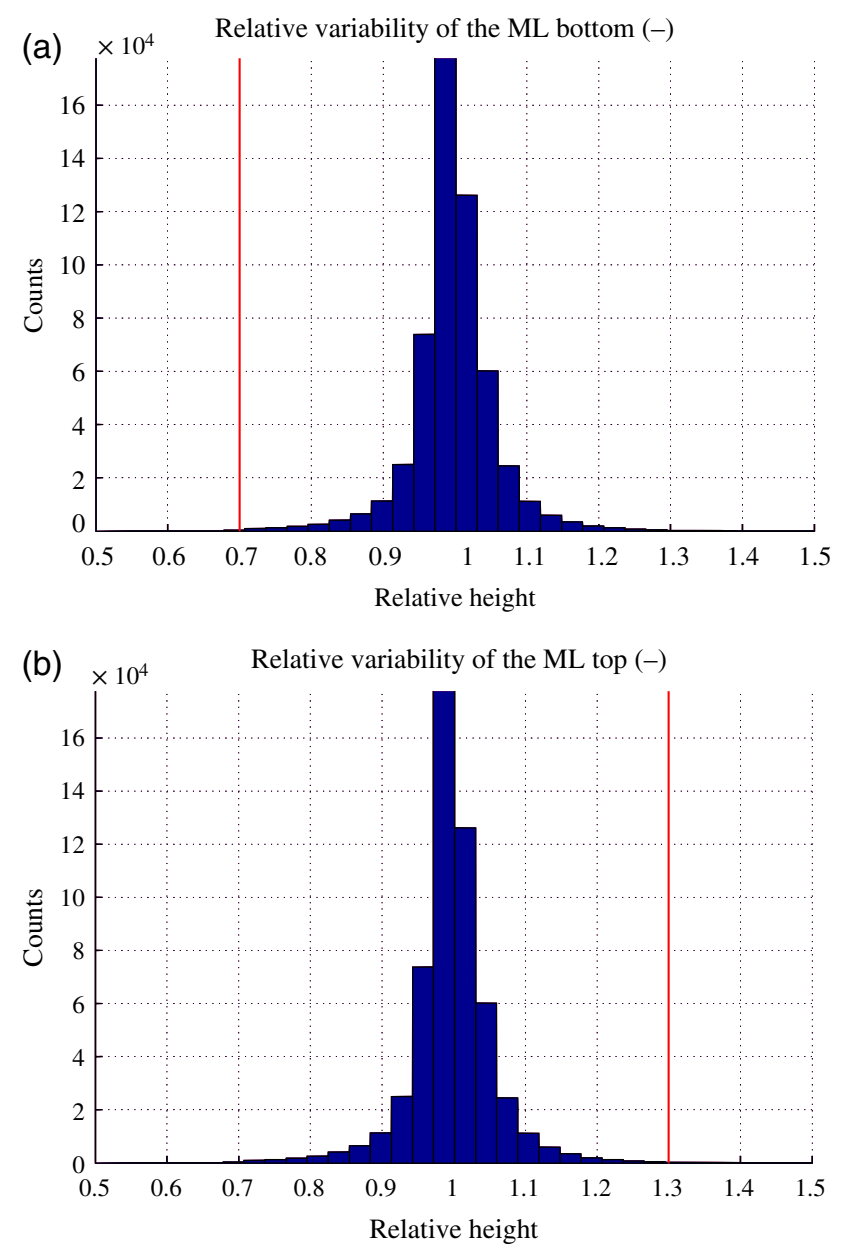

Figure 9. Histogram of relative heights of (a) the top and (b) the bottom of the ML.

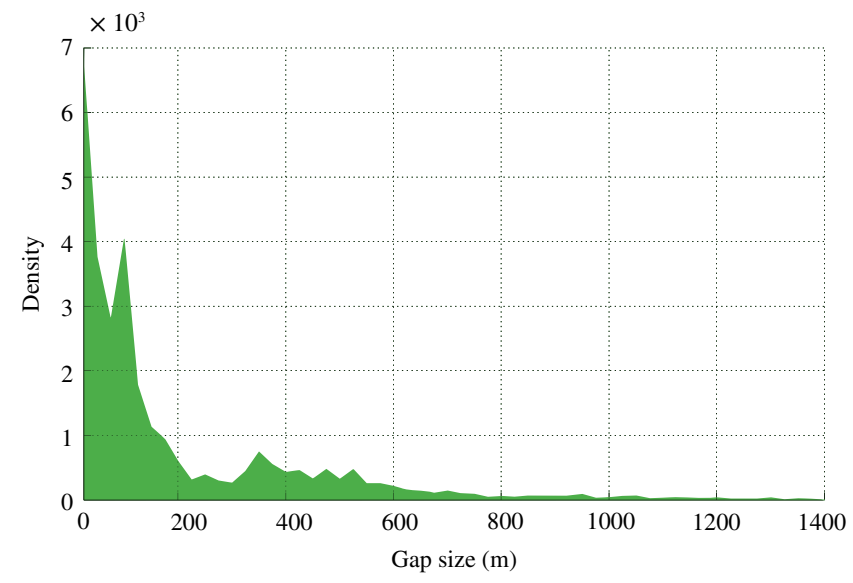

Figure 10. Normalized histogram of the distribution of gap sizes in the detected MLs.

$(<300 \mathrm{~m})$ and can thus be safely interpolated. Accordingly, the recommended value of $L_{\text {gaps, } \max }$ is $250 \mathrm{~m}$.

Interpolation can be useful if the liquid and solid phases have to be discriminated, for example prior to performing a hydrometeor classification. In the context of this work, interpolation was only used in order to get an estimation as complete as possible of the freezing-level height for the hydrometeor classification, but was not used in the characterization of the ML (section 5).

Finally the last parameter $L_{\text {filt,grad }}$ is the length of the moving average filter used to smooth the gradient image, in order to compensate part of the intrinsic noisiness of the gradient. A length of $75 \mathrm{~m}$ is used in practice, which gives a moving window of size $75 \times 75 \mathrm{~m}^{2}$. The size was chosen in order to average the gradient approximatively over one radar bin. The sensitivity of 


\section{Wolfensberger et al.}

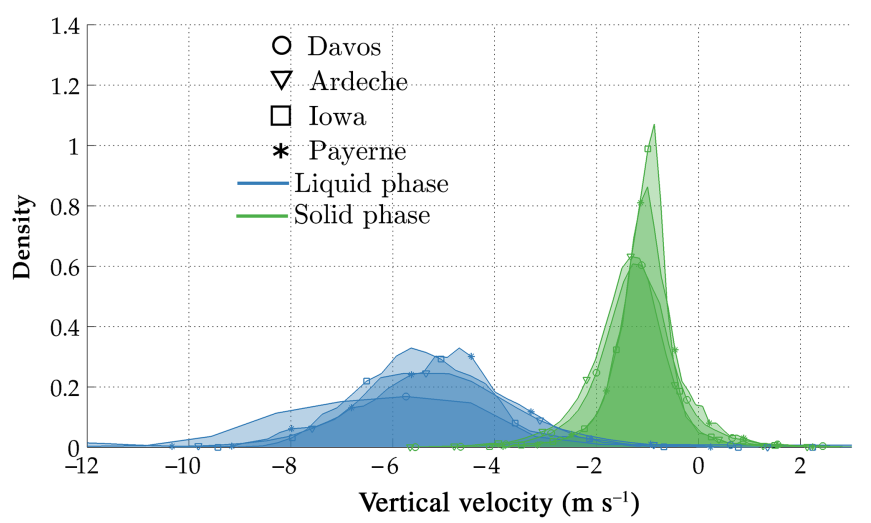

Figure 11. Distributions of the hydrometeor vertical fall velocities in the liquid and solid phases. The different symbols denote the different datasets.

the algorithm to this parameter was tested in a way similar to the gradient threshold. It was observed that doubling the size of the window changes only slightly the output of the algorithm $(85 \%$ of average agreement) and the distributions of the ML thickness (increase of $20 \mathrm{~m}$ in median), while polarimetric signatures stay largely unchanged (variations of less than 1\%). This window size is hence not very critical.

\section{Validation}

\subsection{Vertical hydrometeor fall velocities}

A first assessment of the performance of the algorithm can be conducted by verifying the consistency of its output. The ML is characterized by a change in the hydrometeor fall velocity, with a transition from low velocities in the solid phase to higher velocities in the liquid phase (White et al., 2002). The liquid and solid phases identified by the algorithm should confirm this behaviour.

The empirical probability density functions of hydrometeor vertical fall velocity in the liquid and solid phases, estimated by the radial velocity at $90^{\circ}$ elevation, are shown in Figure 11. The overlapping coefficient, which is a measure of agreement between two distributions and corresponds to the area of overlap (Inman and Bradley, 1989) is respectively 0.06, 0.07, 0.06 and 0.11 for the Payerne, Davos, Iowa and Ardèche datasets, which shows that the distributions within the liquid and solid phases for all datasets are strongly dissimilar. Since hydrometeor fall velocities are independent from the radar variables used as input to the algorithm, this result tends to indicate that the algorithm discriminates well the liquid from the solid phase.

\subsection{Comparison with Payerne radiosoundings}

The Payerne dataset offers a good opportunity to assess the agreement between the output of the algorithm and the freezinglevel height measured by the radiosoundings, assuming that the top of the ML can be associated with the $0^{\circ} \mathrm{C}$ isotherm. To obtain a freezing-level estimation, the algorithm was run with a maximum range of $5000 \mathrm{~m}$ and the detected ML top was averaged over the entire RHI. One difficulty in the comparison is that the distance and the time interval between the sounding and the radar scan can be significant. The geographical distance should not be a major issue since, for the range of isotherm $0^{\circ}$ heights encountered during this campaign $(1500-3000 \mathrm{~m})$, the horizontal advection of the radiosonde is reasonably small, from 3 to $10 \mathrm{~km}$. The time interval is more problematic since soundings are performed only twice daily (at 0000 and 1200 UTC). To deal with this issue, errors were compared when all data were used (interpolating sounding heights linearly through time) and when only radar scans with a maximum time interval of $30 \mathrm{~min}$ to the closest sounding were used. Figure 12 shows that the correspondence is generally good

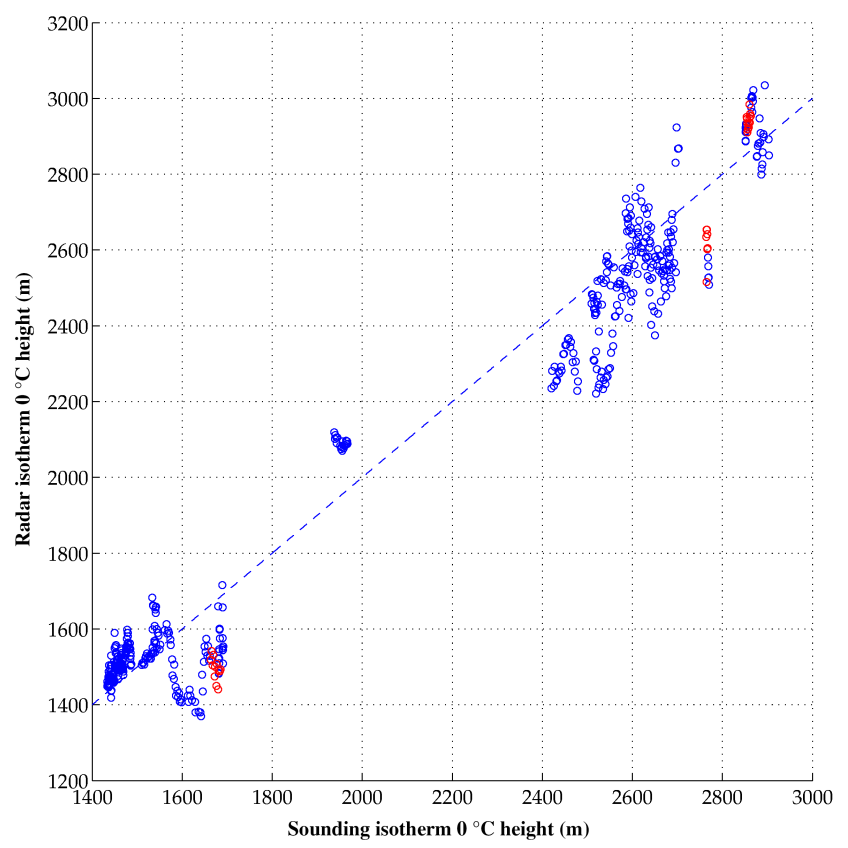

Figure 12. Heights of the $0^{\circ} \mathrm{C}$ isotherm, radar versus soundings, at Payerne. The 1:1 line is shown as dashed blue. Red dots denote radar scans that are separated by at most $30 \mathrm{~min}$ from the closest sounding.

Table 4. Bias and mean absolute error in the radar freezing level estimation for all scans and for scans with a time interval to the radiosounding of maximum 30 min only. The bias is defined as the height of the ML top minus the sounding freezing level height.

\begin{tabular}{lcc}
\hline & Bias $(\mathrm{m})$ & MAE $(\mathrm{m})$ \\
\hline All scans & -55.06 & 94.15 \\
Scan with $\Delta_{T}<30 \mathrm{~min}$ & -80.85 & 130.5 \\
\hline
\end{tabular}

and follows well the 1:1 line with errors rarely exceeding $200 \mathrm{~m}$. This remains true when considering larger time intervals. The average errors are given in Table 4.

The freezing-level height estimated by the algorithm is slightly underestimated but this is still a good agreement considering the radial resolution of the $\operatorname{radar}(75 \mathrm{~m})$ as well as the imperfect matching in time and space. It is worth noticing that the error is larger for small time intervals (red), which can be due to a sampling effect, the number of considered scans being small. Additionally, this also tends to indicate that the effect of the time interpolation is not too large and that freezing-level heights evolve regularly during the day.

\subsection{Comparison with an algorithm adapted from PPI scans}

Most ML detection algorithm are designed for PPI scans of operational radars at C- or S-band (e.g. Brandes and Ikeda, 2004; Giangrande et al., 2008). In order to compare the performance of our algorithm with a more simple approach, we adapted the algorithm of Giangrande et al. (2008) to RHI scans. The adapted algorithm works directly on polar RHI scans and classifies a pixel as belonging to the ML if:

- $\rho_{\mathrm{hv}}>\rho_{\mathrm{hv}, \min }$ and $\rho_{\mathrm{hv}}<\rho_{\mathrm{hv}, \max } ;$

- The maximum of $Z_{\mathrm{H}}$ within a vertical window of $500 \mathrm{~m}$ below and above the pixel is $>30$ and $<47 \mathrm{dBZ}$;

- The maximum of $Z_{\mathrm{DR}}$ within a vertical window of $500 \mathrm{~m}$ above the pixel is $>0.5$ and $<2.5 \mathrm{~dB}$.

The vertical window of $500 \mathrm{~m}$ corresponds to an equivalent range of $500 / \sin \theta \mathrm{m}$, where $\theta$ is the elevation angle. Once the ML has been identified in polar coordinates, it is converted to Cartesian coordinates as in section 2.3.1. 
Table 5. Bias and mean absolute error in the radar freezing level estimation with the modified Giangrande et al. (2008) algorithm for all scans and for scans with a time interval to the radiosounding of maximum $30 \mathrm{~min}$ only.

\begin{tabular}{lcc}
\hline & Bias $(\mathrm{m})$ & MAE $(\mathrm{m})$ \\
\hline All scans & -274.68 & 301.79 \\
Scan with $\Delta_{T}<30 \mathrm{~min}$ & -226.02 & 229.9 \\
\hline
\end{tabular}

Giangrande et al. (2008) recommended using $\rho_{\mathrm{hv}, \min }=0.9$ and $\rho_{\mathrm{hv}, \max }=0.97$, but we chose to use $\rho_{\mathrm{hv} \text {,min }}=0.85$ instead since it is closer to the lower bound of $\rho_{\mathrm{hv}}$ values inside the ML (cf. Figure 7).

Table 5 shows the performance of the modified Giangrande et al. (2008) algorithm on the Payerne dataset from section 4.2. It clearly appears that both the bias and the errors are much larger. The large bias shows that the algorithm underestimates the height of the freezing level because it does not sufficiently take into account the fact that the top of the $\mathrm{BB}$ can be significantly higher than the layer of low $\rho_{\mathrm{hv}}$. The performance on the scans with $\Delta_{T}<30 \mathrm{~min}$ is slightly better but still much worse than for the proposed algorithm (Table 4 ). Note that setting $\rho_{\mathrm{hv} \text {,min }}=0.9$ instead of 0.85 increases slightly the bias and the error (to $-292 \mathrm{~m}$ bias and $316 \mathrm{~m}$ MAE for all scans).

Overall, the designed algorithm accurately detects the freezing level and separates well the liquid and solid phases. It also performs much better than a simpler algorithm originally designed for operational PPI scans and adapted to RHI scans. A benefit of detecting the ML on RHI scans is that the height of its boundaries can be detected all along the radar profile, which allows us to get more information about the geometry and the small-scale variability in shape of the ML.

This new algorithm is a very useful tool in the rest of this work which will focus on the characterization of the melting layer. It can also be used for other purposes, e.g. for comparison with numerical weather models or as a constraint for hydrometeor classification methods.

\section{Characterization of the ML}

All scans from all four available datasets were preprocessed and fed into the ML detection algorithm. Based on the output

Table 6. List of ML descriptors by category.

Geometry
Polarimetry

Doppler

Hydrometeors

\author{
Thickness of the ML (m). \\ Altitudes of top and bottom of the ML (m). \\ Polarimetric variables $\left(Z_{\mathrm{H}}, Z_{\mathrm{DR}}, \rho_{\mathrm{hv}}, K_{\mathrm{dp}}\right)$ \\ in the ML for solid and liquid phases. \\ Bright-band intensity (dBZ). \\ Distance between the maximum of $Z_{\mathrm{H}}$ \\ and the minimum of $\rho_{\mathrm{hv}}(\mathrm{m})$. \\ Gradient of $Z_{\mathrm{H}}$ just above the $\mathrm{ML}\left(\mathrm{dB} \mathrm{m}^{-1}\right)$. \\ Amplitude (with respect to solid phase) \\ of the bright-band peak (dB). \\ Vertical fall speeds in the ML \\ for solid and liquid phases $\left(\mathrm{m} \mathrm{s}^{-1}\right)$. \\ Fractions of aggregates, ice crystals \\ and rimed particles above the ML (-). \\ Thickness of the riming layer $(\mathrm{m})$.
}

of the ML detection algorithm, various ML descriptors were computed, as illustred in Figure 13. They can be grouped into four categories (Table 6). The hydrometeor classification algorithm (section 2.3.3) was used to classify every pixel in the solid phase into one of three classes: aggregates, rimed particles and crystals. The fraction of hydrometeors is simply the fraction of the pixels of one class over all pixels in the solid phase.

\subsection{The ML attenuation effect}

Before focusing on the ML descriptors, the potential error due to the attenuation in the ML was investigated. Attenuation in the ML is a poorly known phenomenon, mainly because its quantification poses many instrumental and methodological problems. Bellon et al. (1997) estimated the attenuation in the ML in the vertical by comparing UHF and X-band radar reflectivity measurements in the solid phase near the echo top assuming that solid hydrometeors at this altitude behave as Rayleigh scatterers. The attenuation effect was increasing with the intensity of the BB and the total attenuation over the entire ML was estimated to be up to $1.7 \mathrm{dBZ}$ for an intensity of $36.5 \mathrm{dBZ}$. At lower elevation angles, the attenuation effect could be even stronger, especially for low melting layers. Klaassen (1990) estimated the attenuation effect in the ML using a new scheme for the calculation of the dielectric

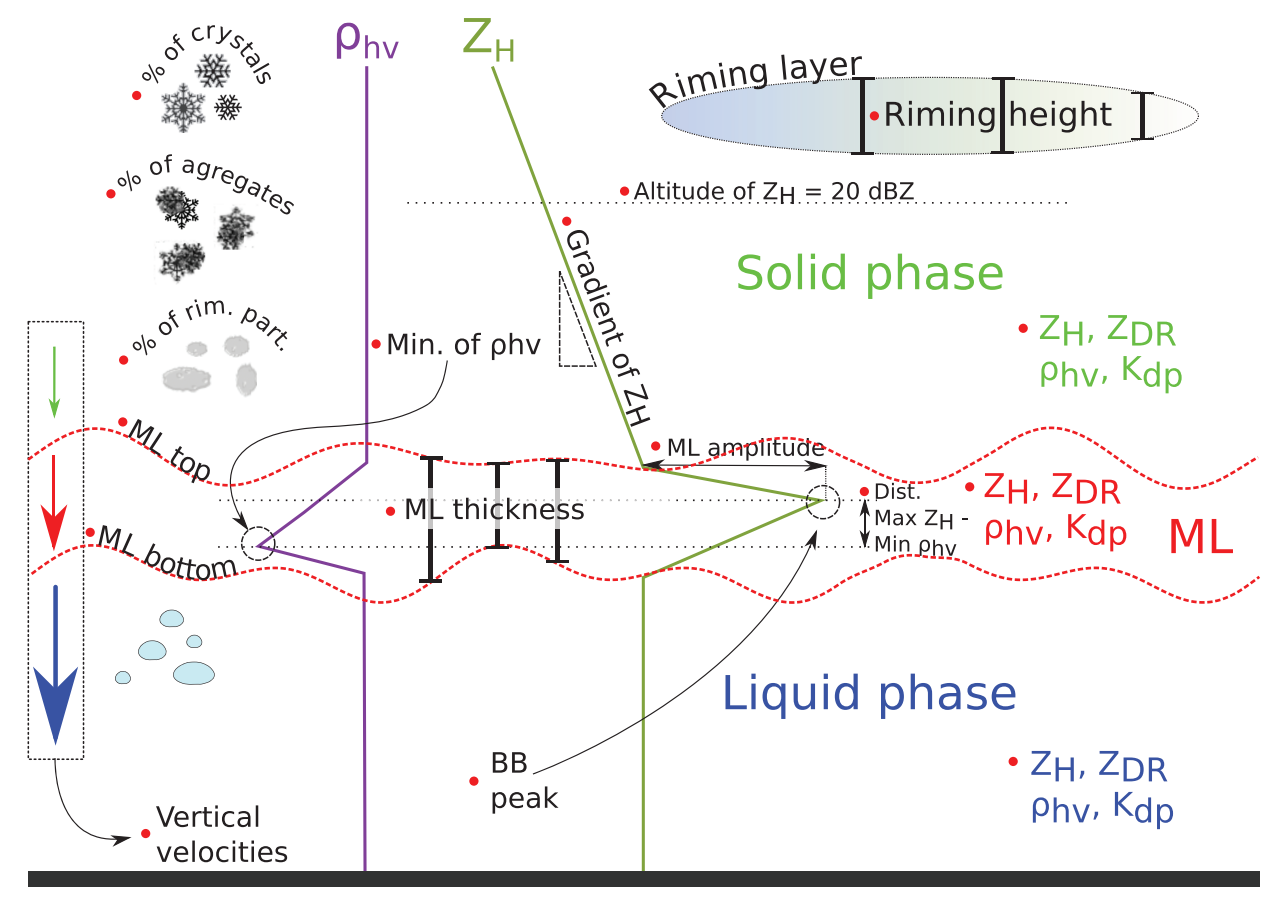

Figure 13. Schematic representation of the computed ML descriptors on a RHI scan. Computed descriptors are highlighted by a red dot. Limits of the ML are shown as red dashed lines. 


\section{Wolfensberger et al.}

properties of melting ice. The estimated specific attenuation effect was variable through the ML, but would reach maximum values of around $1.5 \mathrm{~dB} \mathrm{~km}^{-1}$ at a frequency of $12 \mathrm{GHz}$. Simulations made by Matrosov (2008) gave an average specific attenuation of around $0.3-0.5 \mathrm{~dB} \mathrm{~km}^{-1}$ for rain rates around $2-3 \mathrm{~mm} \mathrm{~h}^{-1}$. Pujol et al. (2012) relied on the simulation of airborne X-band measurements and found much smaller values of attenuation, with a maximum specific attenuation of around $0.2 \mathrm{~dB} \mathrm{~km}^{-1}$. For an average ML thickness of $300 \mathrm{~m}$, this would be 30 times less than Bellon et al. (1997). Additionally, to the author's knowledge, no study has been conducted about the differential attenuation on $Z_{\mathrm{DR}}$ caused by the ML. This effect could also be quite high but is even more difficult to quantify since $Z_{\mathrm{DR}}$ cannot be measured at vertical incidence.

To investigate the bias caused by neglecting the melting-layer attenuation effect, a statistical analysis of the $Z_{\mathrm{H}}$ and $Z_{\mathrm{DR}}$ shift across the melting layer was performed. If the attenuation effect is significant, there should be on average a decrease in $Z_{\mathrm{H}}$ and $Z_{\mathrm{DR}}$ between the point where the beam enters the melting layer (at the bottom) and the point where it leaves the melting layer (at the top). To simplify the notation, we will denote by MLD the distance travelled by the radar beam through the ML. The decrease in $Z_{\mathrm{H}}$ and $Z_{\mathrm{DR}}$ should become more and more important as the MLD increases. For a given radar radial, the MLD depends on both the height of the melting layer and the elevation angle. It is maximal for low elevation angles and low melting layers. It is possible to have a rough idea of the ML attenuation effect by assuming homogeneity of the ML and by computing the shift in the differences of $Z_{\mathrm{H}}$ and $Z_{\mathrm{DR}}$ across the ML with increasing MLD. The validity of this approach is restricted by the assumption of homogeneity, but the very large amount of available data should alleviate the sampling effect. For $Z_{\mathrm{DR}}$, the assumption of homogeneity is more difficult to justify due to the high heterogeneity of particle shapes in the solid phase which can result in a potentially large local variability of the intrinsic (non-attenuated) $Z_{\mathrm{DR}}$. As such, this estimation of the differential attenuation of $Z_{\mathrm{DR}}$ might be biased.

To be consistent with the rest of this work, only the first $5 \mathrm{~km}$ from the radar were considered (section 2.3.1). Results of this analysis are shown as a series of boxplots in Figure 14. A clear, almost linear shift in the distributions of $Z_{\mathrm{DR}}$ differences is visible, whereas in $Z_{\mathrm{H}}$ the shift is less evident and does not vary linearly with the distance. At a MLD of $2300 \mathrm{~m}$, the shift is around $1 \mathrm{~dB}$ in $Z_{\mathrm{H}}$ and $0.6 \mathrm{~dB}$ in $Z_{\mathrm{DR}}$, which corresponds to approximately 16 and $27 \%$ of the local variability, estimated for every distance bin by the $\mathrm{Q}_{90}-\mathrm{Q}_{10}$ interquantile. This observation could give a rough estimation of the ML specific attenuation by dividing the shift by the distance: $0.5 \mathrm{~dB} \mathrm{~km}^{-1}$ for $Z_{\mathrm{H}}$ and $0.37 \mathrm{~dB} \mathrm{~km}^{-1}$ for $Z_{\mathrm{DR}}$.

Matrosov (2008) gave a power law estimating the ML attenuation normalized to the vertical as a function of the rain rate: $A(\mathrm{~dB})=0.048 R^{1.05}$. Using this power law and the classical $Z-R$ relation $Z=200 R^{1.6}$ (Marshall et al., 1955) separately along every radar beam crossing the ML, one can obtain the theoretical total attenuation. Dividing this attenuation for every beam by the sine of the corresponding elevation angle and by the MLD and averaging over all profiles gives an average specific attenuation in $Z_{\mathrm{H}}$ of around $0.2 \mathrm{~dB} \mathrm{~km}^{-1}$, which is smaller than the observed value.

The measured specific $\mathrm{BB}$ attenuation in $Z_{\mathrm{H}}$ can also be compared with measurements by Bellon et al. (1997) who measured the total $\mathrm{BB}$ attenuation at the vertical for some values of $Z_{\mathrm{H}}$ in rain. Interpolating between these measurements and using the same method as before on every radar beam crossing the ML, one obtains an average specific attenuation of around $2 \mathrm{~dB} \mathrm{~km}^{-1}$ which is much larger than our estimation and the estimation of Matrosov (2008). However, one should keep in mind the strong variability from event to event, ${ }^{\ddagger}$ as well as the possibility of non-negligible attenuation in solid precipitation in

Bellon et al. (1997) measured for example $3 \mathrm{~dB}$ for an event with a BB peak of $40 \mathrm{dBZ}$ and then only $0.5 \mathrm{~dB}$ for a nearly identical event data sample two days later. the case of large aggregates. Another possible reason for this large difference comes from the uncertainty of extrapolating the vertical measurements of Bellon et al. (1997) to lower elevation angles.

This shift is small for $Z_{\mathrm{H}}$ compared with the typical values in stratiform rain or in the ML $(20-40 \mathrm{dBZ})$. It is even smaller than the usual calibration error on $Z_{\mathrm{H}}$ which is around $1 \mathrm{dBZ}$. As such, the output of the ML detection algorithm should not be influenced by the ML attenuation effect. Additionally, considering the high values of $Z_{\mathrm{H}}$ in the ML, the effect on the overall distribution of reflectivity in the ML should be limited.

However, the differential attenuation on $Z_{\mathrm{DR}}$ seems to be quite important compared with the usual range of $Z_{\mathrm{DR}}$ values $(0-3 \mathrm{~dB})$. However one should keep in mind that in the solid phase most pixels will have a low MLD and will not be affected very much by attenuation. The third plot of Figure 14 shows that $80 \%$ of all pixels in the solid phase have a MLD smaller than $1300 \mathrm{~m}$. Since correction of this differential attenuation effect is currently not possible, values of $Z_{\mathrm{DR}}$ inside, and to a lesser extent above, the ML should be considered carefully, as they are certainly negatively biased.

\subsection{Polarimetric signature of the $M L$}

The distributions of $Z_{\mathrm{H}}, Z_{\mathrm{DR}}, K_{\mathrm{dp}}$ and $\rho_{\mathrm{hv}}$ for the four datasets are shown in Figure 15. The two derived variables, amplitude of the $\mathrm{BB}$ and distance between peak of $Z_{\mathrm{H}}$ and minimum in $\rho_{\mathrm{hv}}$, are represented as well. In addition, a summary of these distributions as quantiles is given in Table 7. The shapes of the distributions in $\rho_{\mathrm{hv}}$ agree relatively well, but the Iowa and Davos datasets are characterized by the presence of a larger number of smaller values of $\rho_{\mathrm{hv}}$. The shapes of the distributions in $K_{\mathrm{dp}}$ are quite similar for the Davos, Ardèche and Payerne datasets, but the Davos distribution is shifted towards larger $K_{\mathrm{dp}}$. The Iowa dataset differs from the others as its distribution is much more symmetrical with fewer smaller $K_{\mathrm{dp}}$ values. Distributions in $Z_{\mathrm{H}}$ show some discrepancies between datasets. The Ardèche dataset has much stronger $Z_{\mathrm{H}}$ values, whereas the Iowa (IFloodS) dataset has much lower values. The Iowa dataset is quite small and was recorded during a limited period of time (April/May 2014). It is dominated by situations with relatively weak rain rates. In contrast, the Ardèche dataset was recorded in autumn, a season during which very heavy precipitation often occurs over the southeast of France, so part of this discrepancy could be due to this sampling effect. $Z_{\mathrm{DR}}$ distributions generally agree quite well with the exception of the Davos dataset which has stronger values (by around $1 \mathrm{~dB}$ ). Part of this bias could come from the fact that the radar was equipped with flexible waveguides during that campaign, which were later replaced by a rotary joint. This could also explain the shift in $K_{\mathrm{dp}}$ in the Davos dataset.

Giangrande et al. (2008) detected the ML on S-band PPI scans and computed the distributions of $Z_{\mathrm{H}}, Z_{\mathrm{DR}}$ and $\rho_{\mathrm{hv}}$ in wet snow over $29 \mathrm{~h}$ of observation. The measured distribution of $Z_{\mathrm{H}}$ shows a relatively symmetrical distribution with a mode around $30 \mathrm{dBZ}$ whereas the distribution of $Z_{\mathrm{DR}}$ shows a right-skewed distribution with a mode around $1 \mathrm{~dB}$, which is in close agreement with what we observe when merging all datasets. However there is some difference in the distribution of $\rho_{\mathrm{hv}}$ which has a smaller spread and a more symmetrical distribution, with a smaller mode (0.96), but a similar mean. Since the size of their dataset is much smaller than ours, this could be due to a sampling issue.

Additionally, the two derived variables (the BB amplitude and the distance between the peak of $Z_{\mathrm{H}}$ and the minimum in $\rho_{\mathrm{hv}}$ ) have a very similar distribution on all datasets, which tends to show that on average concentration effects and increase of reflectivity due to the BB effect are similar. Durden et al. (1997) computed empirical moments of some ML descriptors over the tropical Pacific region. In terms of $\mathrm{BB}$ intensity (maximum of $Z_{\mathrm{H}}$ ) and amplitude, our overall statistics are in good agreement with their observations as well as the model profiles given by Brandes and Ikeda (2004), with only a few dBZ of difference 31.75 here versus 35.4 (Durden et al., 1997) and 35 (Brandes and Ikeda, 2004). 

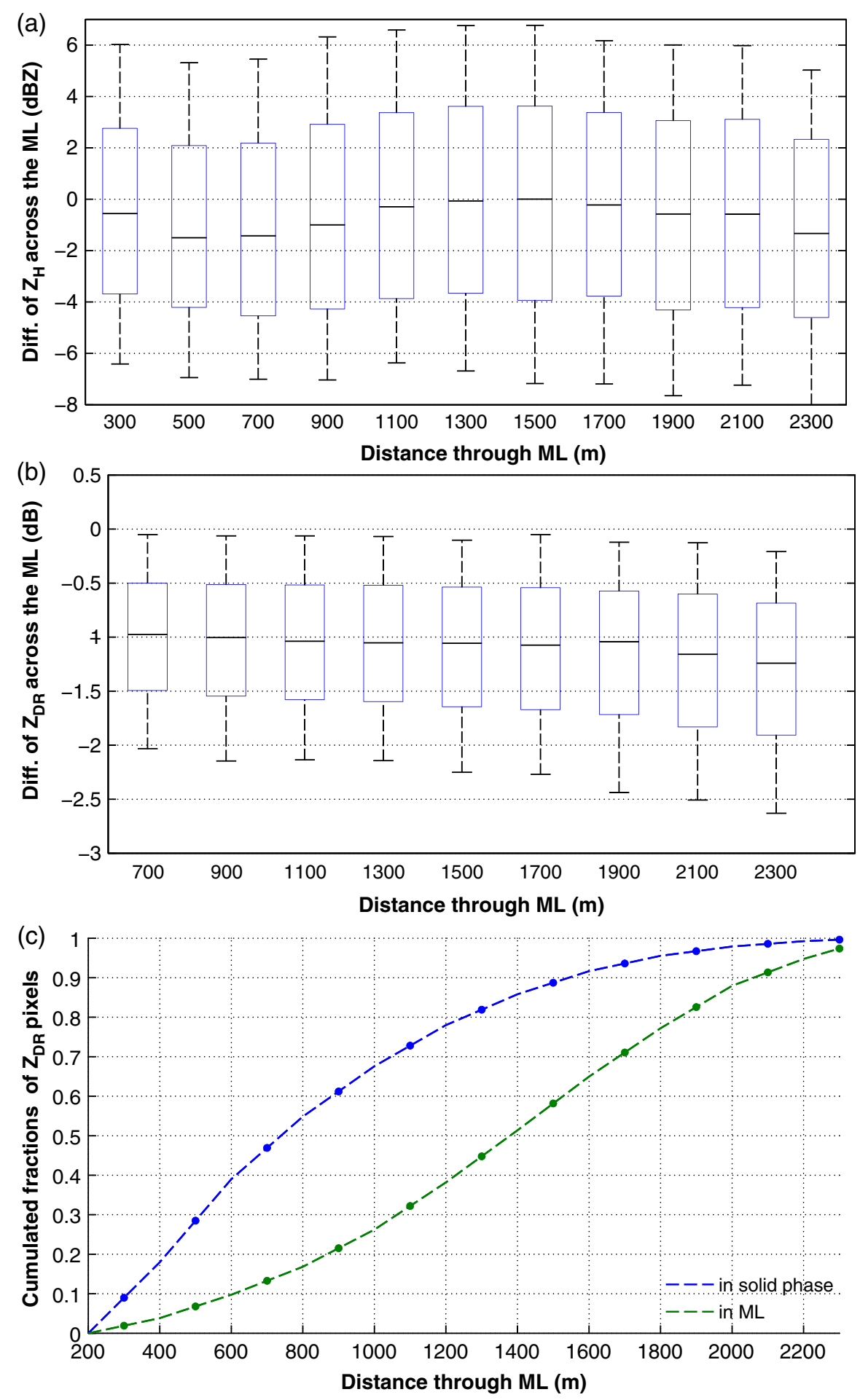

Figure 14. Boxplots of the distributions of the differences in (a) $Z_{\mathrm{H}}$ and (b) $Z_{\mathrm{DR}}$ accross the melting layer and (c) the cumulative fraction of $Z_{\mathrm{DR}}$ pixels for a range of MLDs. Since $Z_{\mathrm{DR}}$ is not reliable at high elevation angles, distributions at short distances are not available. The values in the boxplots are the quantiles 10 (lower whisker), 25, 50, 75 and 90 (upper whisker). Panel (c) gives the fraction of pixels measured in the ML and the solid phase which, in the beam of the radar, have a MLD smaller than the indicated value in abscissa.

Note also that the average minimal value of $\rho_{\mathrm{hv}}(0.86)$ in the ML is equal to the one found by Durden et al. (1997). The distance between the peak of $Z_{\mathrm{H}}$ and the minimum of $\rho_{\mathrm{hv}}$ has an average of $96 \mathrm{~m}$ and a standard deviation of $84 \mathrm{~m}$ which are quite close to those of Durden et al. (1997): 121 and $92 \mathrm{~m}$ respectively. The average vertical velocity in the ML is also very similar (1.22 here versus $1.4 \mathrm{~m} \mathrm{~s}^{-1}$ for Durden et al. (1997)). The good agreement of our observations with those of Durden et al. (1997) indicates again that the ML has very consistent features globally.

In summary, the polarimetric signature of the ML appears to be quite consistent over all datasets, with the exception of $Z_{\mathrm{H}}$ which strongly depends on the intensity of the recorded rainfall events. Additionally, the distribution of both the BB amplitude and the distance between the maximum in $Z_{\mathrm{H}}$ and the minimum in $\rho_{\mathrm{hv}}$, which is related to concentration effects, are also very similar for all the considered climatic regions.

\subsection{Vertical profiles of polarimetric variables through the $M L$}

The polarimetric variables are not uniform within the ML and exhibit a vertical structure. Figure 16 shows the distributions of $Z_{\mathrm{H}}, Z_{\mathrm{DR}}$ and $\rho_{\mathrm{hv}}$ as a function of the relative height inside the ML ( 0 corresponds to the bottom and 1 to the top of the detected ML). Note that $K_{\mathrm{dp}}$ is not represented because it shows no significant dependence on height. It can be seen that the height of the peak in $Z_{\mathrm{H}}$ (maximum of the $\mathrm{BB}$ ) is around $25 \%$ higher than the minimum in $\rho_{\mathrm{hv}}$. The ML also shows a peak in $Z_{\mathrm{DR}}$ in the lower 


\section{Wolfensberger et al.}
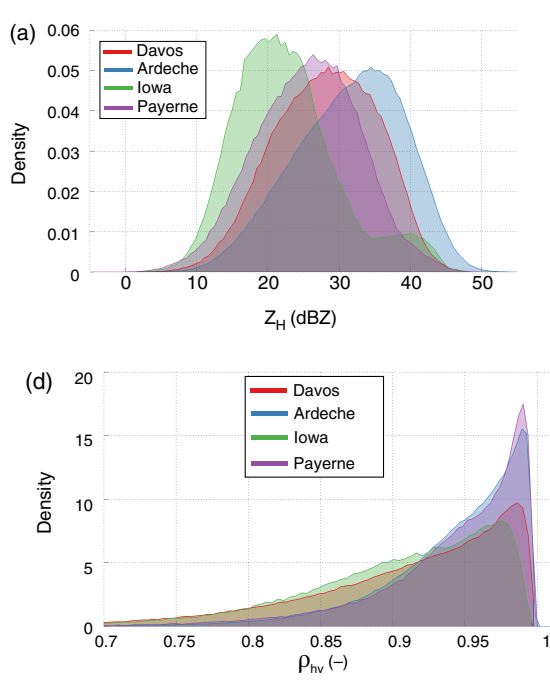
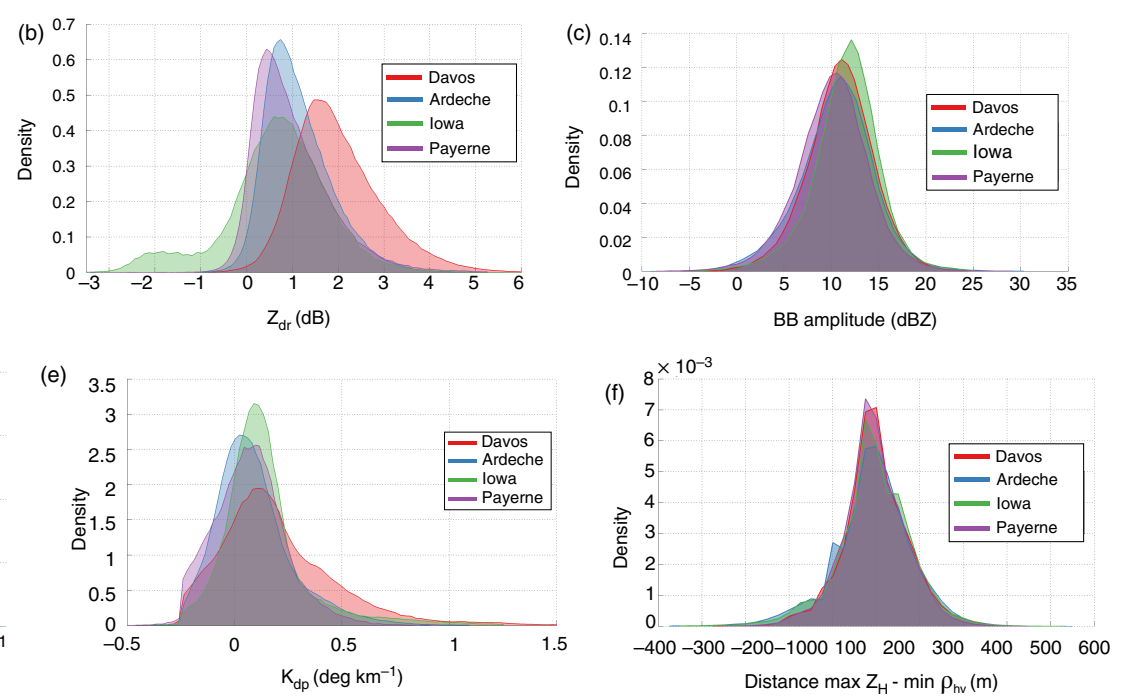

Figure 15. Polarimetric signatures within the ML: (a) $Z_{\mathrm{H}}$, (b) $Z_{\mathrm{DR}}$, (c) amplitude of $Z_{\mathrm{H}}$ (between solid phase and ML), (d) $\rho_{\mathrm{hv}}$, (e) $K_{\mathrm{dp}}$ and (f) distance between peak in $Z_{\mathrm{H}}$ and minimum in $\rho_{\mathrm{hv}}$.

Table 7. Statistics describing the distributions of the polarimetric variables within the melting layer. The bright-band (BB) intensity is simply the maximum of $Z_{H}$ in every vertical column of the ML.

\begin{tabular}{|c|c|c|c|c|c|c|}
\hline Variable & Stat. & All & Davos & Ardèche & Iowa & Payerne \\
\hline \multirow[t]{4}{*}{$Z_{H}$} & (a) & 29.04 & 28.10 & 31.44 & 22.67 & 25.59 \\
\hline & (b) & 7.97 & 7.14 & 7.62 & 7.45 & 7.24 \\
\hline & (c) & 18.28 & 18.54 & 20.88 & 14.06 & 16.03 \\
\hline & (d) & 39.34 & 37.33 & 40.92 & 32.78 & 34.69 \\
\hline \multirow[t]{4}{*}{ BB peak } & (a) & 31.75 & 31.01 & 34.02 & 25.46 & 29.00 \\
\hline & (b) & 7.35 & 6.39 & 7.20 & 6.82 & 6.33 \\
\hline & (c) & 21.81 & 22.40 & 23.95 & 17.15 & 20.48 \\
\hline & (d) & 41.43 & 39.38 & 42.99 & 33.51 & 37.00 \\
\hline \multirow{4}{*}{$Z_{D R}$} & (a) & 1.99 & 1.99 & 1.13 & 0.65 & 0.98 \\
\hline & (b) & 0.95 & 0.95 & 0.72 & 1.14 & 0.83 \\
\hline & (c) & 0.30 & 0.92 & 0.33 & -0.77 & 0.12 \\
\hline & (d) & 2.55 & 3.26 & 2.11 & 1.95 & 2.09 \\
\hline \multirow[t]{4}{*}{$\rho_{h v}$} & (a) & 0.93 & 0.91 & 0.94 & 0.91 & 0.94 \\
\hline & (b) & 0.06 & 0.07 & 0.05 & 0.07 & 0.05 \\
\hline & (c) & 0.85 & 0.81 & 0.88 & 0.81 & 0.87 \\
\hline & (d) & 0.99 & 0.98 & 0.99 & 0.98 & 0.99 \\
\hline \multirow[t]{4}{*}{ Min.of $\rho_{h v}$} & (a) & 0.86 & 0.82 & 0.89 & 0.82 & 0.88 \\
\hline & (b) & 0.07 & 0.07 & 0.05 & 0.06 & 0.06 \\
\hline & (c) & 0.76 & 0.71 & 0.81 & 0.73 & 0.79 \\
\hline & (d) & 0.94 & 0.90 & 0.94 & 0.89 & 0.94 \\
\hline \multirow[t]{4}{*}{$K_{d p}$} & (a) & 0.11 & 0.20 & 0.09 & 0.14 & 0.07 \\
\hline & (b) & 0.21 & 0.28 & 0.18 & 0.21 & 0.17 \\
\hline & (c) & -0.11 & -0.10 & -0.11 & -0.06 & -0.15 \\
\hline & (d) & 0.38 & 0.55 & 0.33 & 0.37 & 0.28 \\
\hline
\end{tabular}

(a) = Mean; (b) =St.Dev.; (c) =Q10\%; (d) = Q90\%.

part of the ML at the same height as the minimum in $\rho_{\mathrm{hv}}$. The lower heights of the peaks of $Z_{\mathrm{DR}}$ and $\rho_{\mathrm{hv}}$ can be explained by the fact that, unlike $Z_{\mathrm{H}}$, these two radar variables are insensitive to concentration effects. The decrease in $Z_{\mathrm{DR}}$ near the bottom of the ML could be due to the break-up of large melted aggregates. However, one should keep in mind the differential attenuation effect of the ML on $Z_{\mathrm{DR}}$ which could also contribute to the lower height of the $Z_{\mathrm{DR}}$ peak.

\subsection{Geometry of the $M L$}

\subsubsection{Thickness}

The detected MLs have on average a very similar geometry on all datasets. Figure 17(a) shows the distribution of the ML thickness; all distributions have a similar shape with a strong mode around $300 \mathrm{~m}$, and a long right tail. The melting layer in Payerne is slightly thinner (purple area) but the differences are small relative to the radial resolution. Generally differences in the mean are small (maximum $35 \mathrm{~m}$ ) and quantiles also agree well between datasets; the quantile $10 \%$ is around $250 \mathrm{~m}$ whereas the quantile $90 \%$ is always around $450 \mathrm{~m}$. This suggests that on average the thickness of the ML is independent of the climatic conditions and the topography. It can be observed that the thickness of the ML never gets below $175 \mathrm{~m}$ but can reach values up to $600 \mathrm{~m}$. The minimal thickness is probably linked with the minimal time snowflakes need to completely melt. The time required for complete melting can be roughly estimated for every RHI scan by dividing the thickness of the ML by the vertical velocity. This gives an average time (over all available scans) of about 2 min for particles to melt completely.

Our observations of the ML thickness agree well with other observations made in the literature. The distribution of ML thickness observed by Giangrande et al. (2008) looks very similar with a marked right tail and a mode around $300 \mathrm{~m}$. Bandera et al. (1998) used a similar ML detection algorithm to process $200 \mathrm{RHI}$ scans recorded over the UK and observed an average thickness of $300 \mathrm{~m}$ which is very close to our observed average value $(320 \mathrm{~m})$. Durden et al. (1997) found a slightly larger average thickness of $400 \mathrm{~m}$, though considering the radial resolution of the radar $(75 \mathrm{~m})$ this difference is barely significant. One possible explanation is that their estimation of the ML is based solely on the detection of the $\mathrm{BB}$ whereas on the current algorithm $\rho_{\mathrm{hv}}$ is also considered for the detection of the base of the ML. In the case of strong precipitation, the lower part of the $\mathrm{BB}$ is not as well defined as the upper part and this could lead to a slightly larger thickness of the ML.

\subsubsection{Horizontal variability}

The horizontal variability of the ML can be quantified by the variograms of the ML thickness and of the heights of the top and bottom of the ML. The variogram is a function that gives half of the average squared difference between pairs of points separated by a given distance (Chilès and Delfiner, 1999). This gives indication about the decorrelation distance, ${ }^{\S}$ the sub-grid variability and the smoothness of a process. The beam-broadening effect causes an artificial trend in the thickness and boundaries of the ML with increasing distance from the radar. To alleviate this effect, the variograms were computed for every scan on linearly detrended 

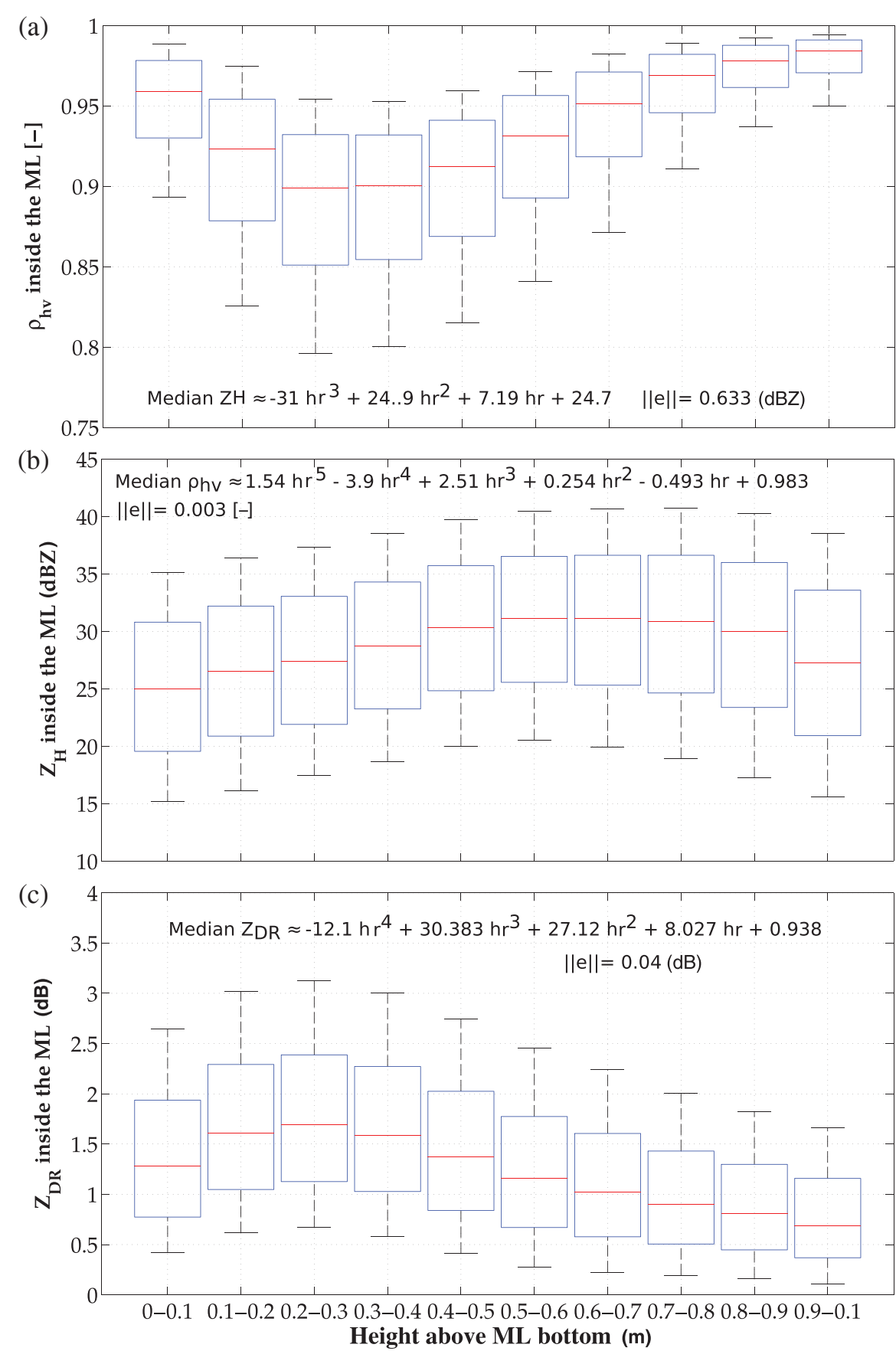

Figure 16. Boxplots of the distributions of (a) $Z_{\mathrm{H}}$, (b) $\rho_{\mathrm{hv}}$ and (c) $Z_{\mathrm{DR}}$ as a function of the relative height inside the ML. Least-square fitting polynomials of the medians as a function of relative height $h_{r}$ are also shown, along with their associated norm of residuals $\|e\|$.

variables. Additionaly, to account for the fact that datasets are of different sizes, the variograms were normalized, i.e. divided by the corresponding variances (of ML thickness or top and bottom ML heights). For every dataset, the variograms were then averaged over all scans.

The normalized variograms of the ML boundaries and thickness (Figure 17(b)) show a similar structure between the datasets, with a similar range and a similar slope especially at low distances, where the variability is greatest. The ML top boundary reaches decorrelation at around over $1500 \mathrm{~m}$, whereas the bottom of the ML seems to be smoother and does not decorrelate completely over $2500 \mathrm{~m}$. Experiments show that this is mainly due to the use of only $Z_{\mathrm{H}}$ to detect the top of the ML. Indeed, unlike $\rho_{\mathrm{hv}}, Z_{\mathrm{H}}$ is dependent on the concentration of hydrometeors and is more strongly influenced by large hydrometeors. The variogram of the thickness has a similar trend but with an even smaller decorrelation range. This can be due to the fact that, after detrending, heights of the top and bottom of the ML are positively yet not totally correlated $(r=0.62)$.

\section{Correlation analysis of ML descriptors}

\subsection{Factors controlling the ML thickness}

Although the ML has a quite consistent shape on average, variations of the ML thickness can be quite significant between and especially during precipitation events. This can be seen for example on the HyMeX dataset (Figure 18), where the ML thickness can easily vary from 250 to $500 \mathrm{~m}$ within the same precipitation event.

In order to identify the possible causes of increased thickness of the ML, a correlation analysis of all ML variables was performed. Before calculating the correlations, the ML statistics described in Table 6 were averaged over every single RHI scan in order to reduce them to the same dimension. ${ }^{9}$ Before computing the

'All ML descriptors are not defined in the same domain, for example the vertical velocity is only available at the vertical whereas $Z_{\mathrm{DR}}$ is available only at low elevation angles. 

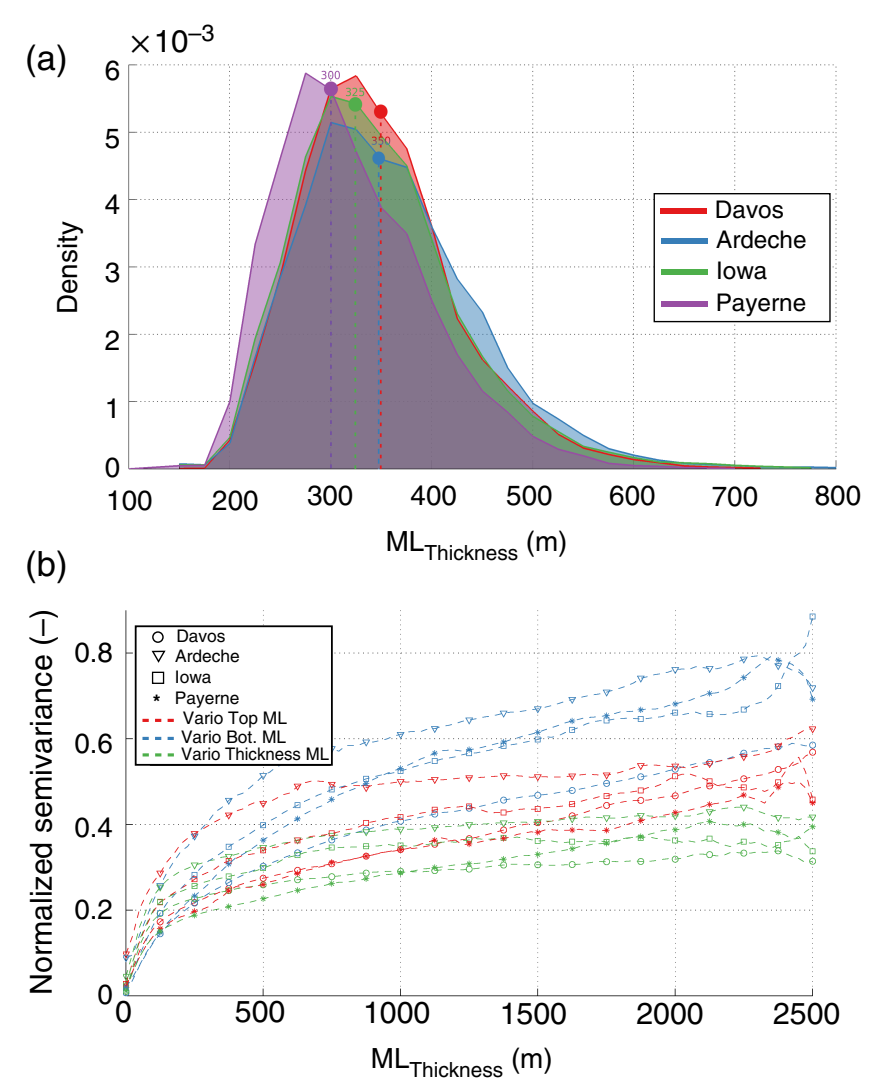

Figure 17. (a) Distributions of the ML thickness for the four datasets, with medians indicated by dashed lines. (b) Normalized (by the variance) variograms of the ML thickness and top and bottom heights. The $x$-axis is the horizontal distance along the RHI.

correlations, the variables were also log-transformed to account for possible nonlinearities.

First of all, it is interesting to notice that there is no correlation between the altitude of the top or bottom of the ML and the thickness of the ML $(r=0.08$ for the top and $r=-0.013$ for the bottom). Consequently, the seasonal variability of the ML, characterized mostly by variations in the freezing-level height, does not seem to contribute significantly to the variability in the ML thickness.

However, other descriptors have quite high correlations with the ML thickness. These relations are shown in the form of a correlation plot in Figure 19. Unsurprisingly, the ML thickness depends strongly on the intensity of $Z_{\mathrm{H}}$ in the ML $(r=0.77)$. Such a correlation was also observed by Durden et al. (1997). The clear linear trend between the two variables is shown in Figure 20. The ML thickness is also strongly correlated with $Z_{\mathrm{H}}$ in the liquid phase $(r=0.72)$. A higher reflectivity in the liquid phase indicates a higher rain rate which corresponds to a larger mass of ice to melt, hence an increase in the ML thickness. This strong correlation also shows that by simply knowing the reflectivity in rain, one can already get some relevant information about the properties of the ML. The intensity of $Z_{\mathrm{H}}$ in the ML and the thickness are also strongly related to the vertical extension characterized by the distance of the $20 \mathrm{dBZ} Z_{\mathrm{H}}$ contour from the ML $(r=0.9$ and $r=0.7$ respectively). A possible explanation is the fact that intense BBs are usually associated with higher $Z_{\mathrm{H}}$ in the solid phase, due to the presence of larger hydrometeors. $K_{\mathrm{dp}}$ in the ML is slightly less correlated with the thickness $(r=0.42)$, but considering the large amount of data this is still an important correlation. Note that $K_{\mathrm{dp}}$ is not redundant with $Z_{\mathrm{H}}$, since their correlation is relatively low $(r=0.36)$. Additionally, the thickness is also well correlated with the vertical fall velocity in the ML $(r=0.61)$. This could be due to two reasons: indirectly because dense particles, which take longer to melt, have also higher fall speeds, and directly because fast falling particles will travel further before melting and will extend the ML downwards.
Another important factor is the gradient of $Z_{\mathrm{H}}$ above the ML, which is positively correlated with the ML thickness $(r=0.57)$; thicker melting layers are associated with a faster decrease in reflectivity above the melting layer. This is possibly the case when the reflectivity in the solid phase is relatively high due to the presence of denser and larger solid particles. Fabry and Zawadzki (1995) also observed such a correlation and suggested that in the case of high rainfall rates, updraughts could be strong enough to bring considerable amounts of cloud water from below the ML into the solid phase above, resulting in 'particularly wet graupel particles', with a high reflectivity. To verify this hypothesis, the relation between the thickness of the ML and the spectral width within the ML both taken at vertical incidence was studied. However, no strong correlation was detected $(r=0.18)$.

A thicker ML is quite often associated with the presence of a layer of rimed particles above the ML ( $r=0.56$ with the thickness of the riming layer). This could be due to the higher density of these particles hence the increasing time it takes for them to melt as well as their larger fall velocities (Pruppacher and Klett, 1997). In the same way, a thicker ML is also correlated with a smaller fraction of ice crystals, since ice crystals and rimed particles are negatively correlated $(r=-0.67)$. The distance between the peak in $Z_{\mathrm{H}}$ and the minimum in $\rho_{\mathrm{hv}}$ is also positively correlated to the thickness $(r=0.44)$ which indicates that the concentration of hydrometeors also seems to play a role. The linear trend between the two variables is visible in Figure 20. Indeed, when the concentration of hydrometeors is higher, the shift in altitude between the peak in $Z_{\mathrm{H}}$ and the minimum in $\rho_{\mathrm{hv}}$ increases, since $Z_{\mathrm{H}}$ is sensitive to concentration effects but $\rho_{\mathrm{hv}}$ is not. An increased hydrometeor concentration could lead to an increase in the diabatic cooling of the surrounding air during the melting process. This effect can be quite important when the situation is very stable and when horizontal temperature advections are small (Kain et al., 2000). The cooling effect increases with the precipitation intensity and can significantly lower the freezing level. Note also that the minimum in $\rho_{\mathrm{hv}}$ in the ML is less correlated than $Z_{\mathrm{H}}$ with the ML thickness $(r=-0.44)$. Both variables are only weakly correlated $(r=-0.26)$. The differences between these two variables, computed after normalizing them by the mean, are significantly correlated with the vertical distance between the peak of reflectivity and the minimum of $\rho_{\mathrm{hv}}$ inside the ML $(r=-0.59)$, which seems to indicate that strong concentrations of solid hydrometeors above the ML can lead to a decoupling of $Z_{\mathrm{H}}$ and $\rho_{\mathrm{hv}}$ within the ML.

According to Durden et al. (1997), the cooling effect can increase the thickness of the ML since particles will take more time to melt. Note that all factors described above, with the exception of the BB amplitude, are statistically significantly correlated with the ML thickness (at $\alpha=1 \%$ ).

Finally, it can seem surprising that the amplitude of the BB does not seem to be correlated with the ML thickness, nor the intensity of $Z_{\mathrm{H}}$ in the ML. This can be explained by the high correlation between $Z_{\mathrm{H}}$ in the ML and $Z_{\mathrm{H}}$ in the solid phase $(r=0.8)$. Small amplitudes of the ML can be caused either by a weak stratiform situation with a thin ML, where the flux is small, or by a strong stratiform situation with high reflectivity above the ML (aggregates and/or rimed particles). In fact, the relation between the amplitude of $Z_{\mathrm{H}}$ in the ML and the thickness of the ML seems to be weakly quadratic. When considering only MLs with a thickness larger than the median (>350 m), the correlation becomes negative $(r=-0.38)$, which shows that thick MLs tend to be associated with a smaller amplitude between the BB peak and the reflectivity in the solid phase. The correlation becomes positive $(r=0.25)$ when considering only MLs with a thickness smaller than the median.

Finally, unlike Durden et al. (1997), we did not identify a significant correlation between altitude of the ML and intensity of the BB $(r=-0.06)$. This might be due to the fact that, unlike in tropical regions, variations in the ML height in temperate climate are dominated by seasonal variations. 

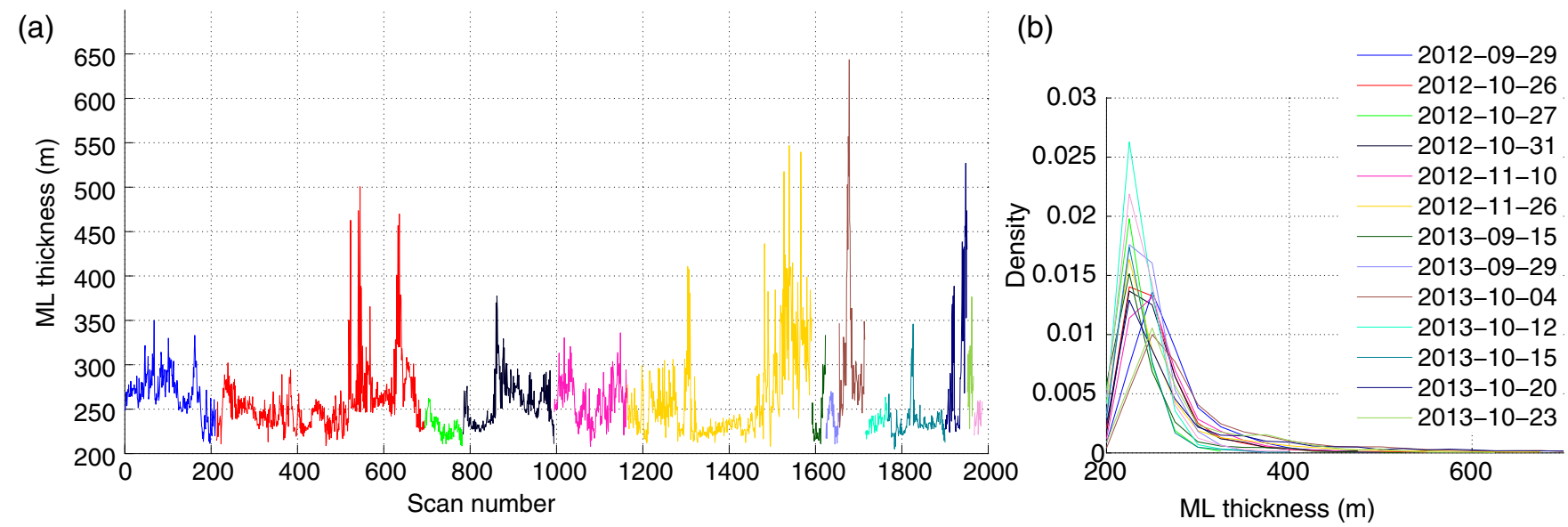

Figure 18. Event by event variability of the ML thickness on the HyMeX dataset. Each coloured line denotes a different precipitation event. (a) Timeline showing the mean ML thickness for every scan. For visualization purposes, all lines are displayed adjacently even if they are in fact temporally separated. (b) The distributions of the ML thickness within all precipitation events.

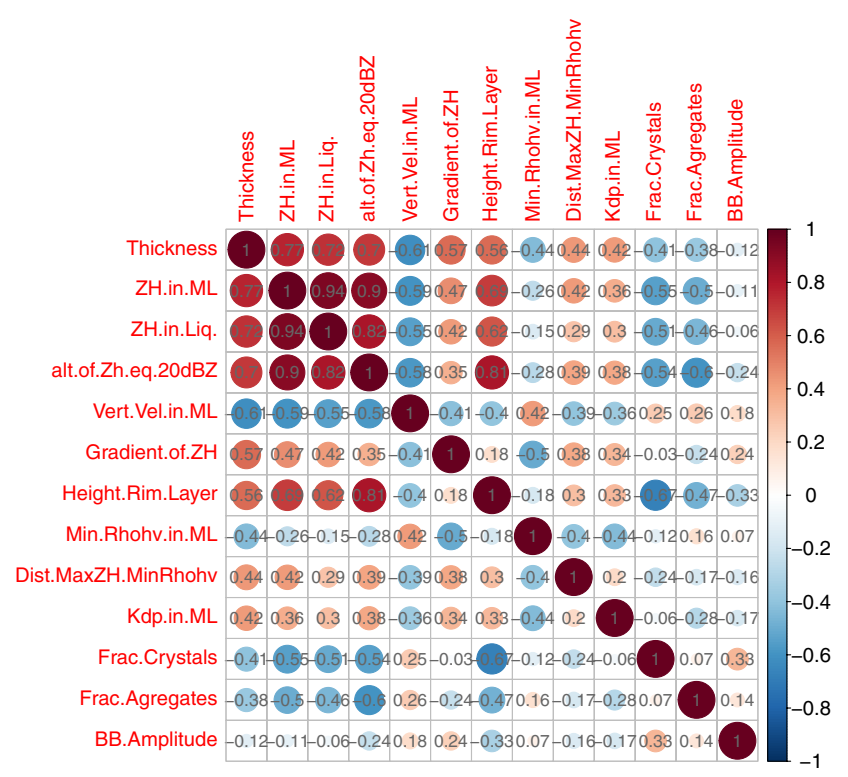

Figure 19. Correlation plot of some relevant variables. Positive correlations are in red and negative correlations in blue.

\subsection{Principal component analysis}

Principal component analysis (PCA) is a statistical procedure that tries to explain the covariance structure of data by means of a small number of orthogonal components which are linear combinations of the original variables. Transformation is done in such a way that the components are ordered by decreasing variance and the first components explain the largest part of the variability in the data.

To gain a better understanding of the relations between the selected factors, a PCA on standardized data was performed. The PCA was performed with ROBPCA, a robust version of the algorithm (Hubbert et al., 2005), that does not rely on the empirical covariance matrix. To simplify the analysis, only the first two components were kept. They explain respectively 52 and $24 \%$ of the total variance. The biplot representing the factors projected into the space of the components is shown in Figure 21. The arrows represent the loading which is the weight by which every standardized variable should be multiplied to get the component scores. Their length is proportional to the contribution of the variable to the two components and the angle between two arrows is an approximate measure of their correlation. It can be seen that the first component corresponds mostly to the intensity and vertical extension of the $\mathrm{BB}$, the presence of riming and the thickness. The fraction of rimed particles has a similar
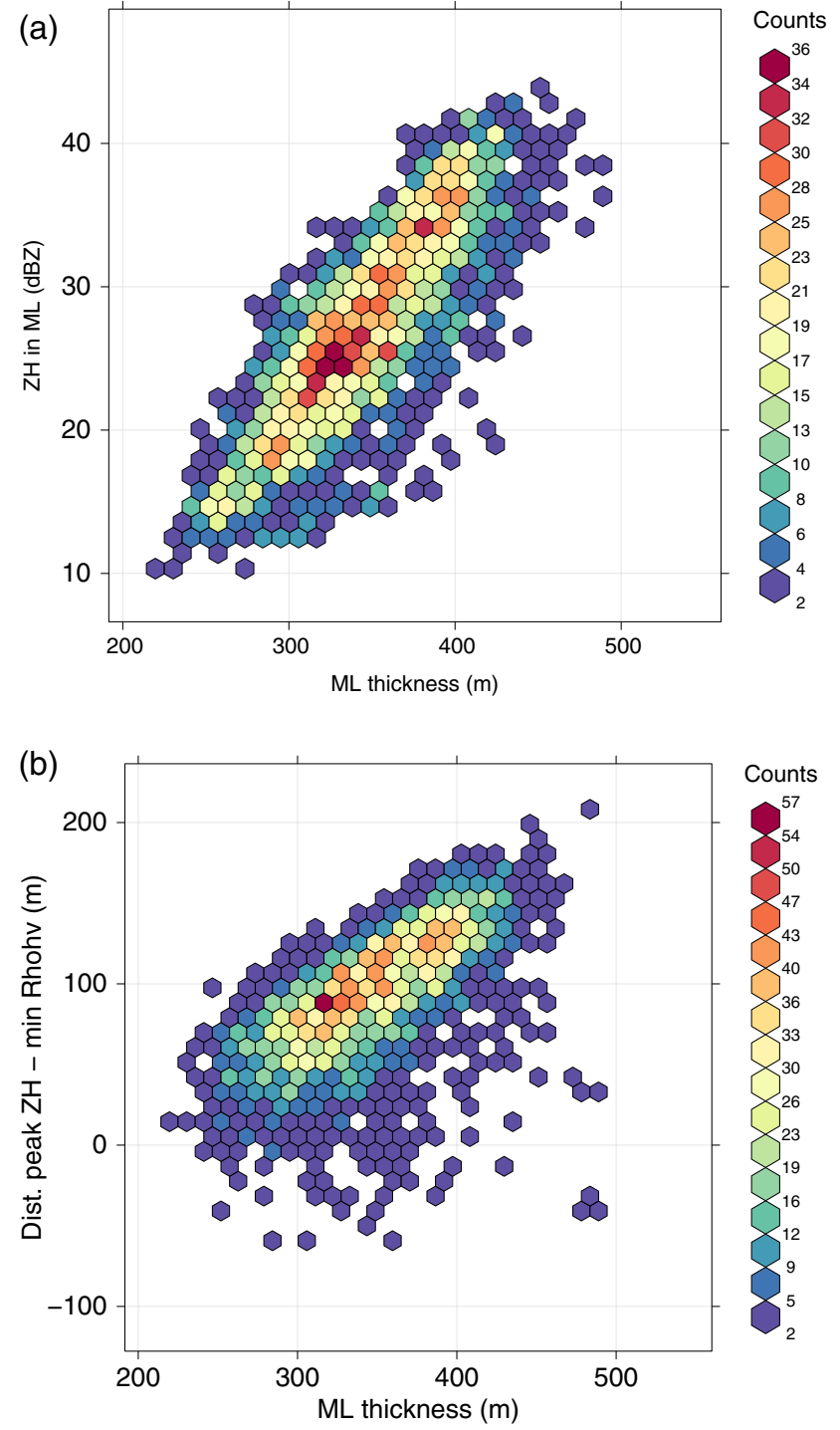

Figure 20. Scatterplots of (a) thickness versus $Z_{\mathrm{H}}$ in the ML and (b) thickness versus distance between peak of $Z_{\mathrm{H}}$ and minimum of $\rho_{\mathrm{hv}}$. Counts indicate the number of points in every hexagonal bin.

and significant contribution to both components. The second component depends mostly on the signature in $\rho_{\mathrm{hv}}$ and $Z_{\mathrm{DR}}$ in the ML. Considering their loading and the relative weight of every component, it can be seen that the most important factors explaining the variability in the ML are (in order of importance) the fraction of riming, the gradient of $Z_{\mathrm{H}}$ in the solid phase and 


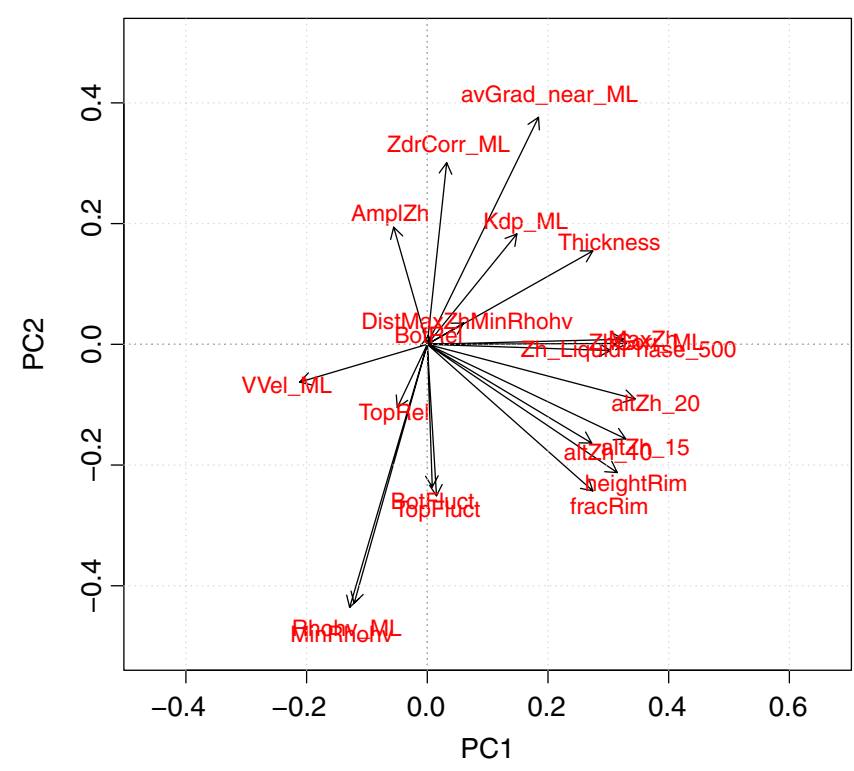

Figure 21. Biplot of the ML descriptors projected onto the plane of the first two PCA components. The first two components explain $60 \%$ of the total variance. The 'Corr' subscript indicates that the variable $\left(Z_{\mathrm{H}}\right.$ or $\left.Z_{\mathrm{DR}}\right)$ has been corrected for attenuation in the liquid phase.

the signature in $\rho_{\mathrm{hv}}$ of the ML. The thickness of the ML depends both on the signature in $\rho_{\mathrm{hv}}$ and on the signature in $Z_{\mathrm{H}}$, which correspond well to the first and second components; as such its variability is a good indicator of the overall variability of the ML. As shown in the correlation analysis, the thickness is also closely related to the vertical velocities inside and below the ML.

\section{Summary and conclusion}

In this work, a new algorithm was developed to automatically detect the melting layer (ML) on polarimetric RHI scans. This algorithm was then used to characterize the ML on highresolution RHI scans collected by X-band polarimetric radars in different climatic regions.

The ML detection algorithm is based on the identification of strong vertical gradients in the reflectivity factor at horizontal polarization $Z_{\mathrm{H}}$ and the copolar cross-correlation coefficient $\rho_{\mathrm{hv}}$. The algorithm takes RHI scans projected to Cartesian coordinates as input and is divided into two parts: in the first part the bottom and the top of the melting layer are detected using both $Z_{\mathrm{H}}$ and $\rho_{\mathrm{hv}}$, while in the second part the estimation of the ML top is refined by using only $Z_{\mathrm{H}}$.

The algorithm was validated by comparing the height of the top of the detected ML with freezing level measurements from collocated radiosoundings. The freezing level estimation of the algorithm was shown to be accurate, with an average error close to the radial resolution of the radar and only a small negative bias. In addition, the distributions of the vertical fall velocities were compared between the liquid phase (below the detected melting layer) and the solid phase (above). The distributions were clearly separated with almost no overlap, showing that the algorithm separates well the liquid and the solid phases.

The shift in $Z_{\mathrm{DR}}$ and $Z_{\mathrm{H}}$ across the ML was analyzed in order to get a rough idea of the attenuation effect of the ML. It was shown that the effect on $Z_{\mathrm{DR}}$ could be important and outweighs by far the attenuation effect caused by liquid statiform rain, whereas the effect on $Z_{\mathrm{H}}$ was only small.

The ML detection algorithm was used to characterize the ML in terms of polarimetric and geometric signatures on four large datasets of radar scans from different topographic and climatic regions (south of France, Swiss Alps, Swiss plains and Iowa). Additionally, a hydrometeor classification was performed in the solid phase above the ML. The thickness of the ML was shown to be on average very similar on all datasets, with a slightly right skewed distribution and a mean between 300 and $330 \mathrm{~m}$. Similarly, the horizontal variability of the thickness and the relative heights of the ML boundaries, characterized by their variograms, showed very similar decorrelation ranges and slopes between datasets.

In terms of polarimetric signature, the ML had similar distributions in $K_{\mathrm{dp}}, \rho_{\mathrm{hv}}$ and $Z_{\mathrm{DR}}$ with the exception of the dataset from Davos in the Swiss Alps which had higher values of $Z_{\mathrm{DR}}$. This could be due to the use of different radar waveguides. Differences in $Z_{\mathrm{H}}$ were more important, with variations in the mean ranging from 2 to $8 \mathrm{dBZ}$ between datasets. Average values of the peak of $Z_{\mathrm{H}}$, the minimum in $\rho_{\mathrm{hv}}$ and the vertical distance separating them were found to be in good agreement with observations made by Durden et al. (1997) over the Pacific tropical region.

Even though the geometrical signature of the ML is quite homogeneous on average, it was shown to be quite variable during and between precipitation events. To gain a better understanding of this variability a correlation analysis between descriptors of the ML was carried out. Results indicate that a thick ML is usually associated with a strong bright band, a higher vertical extension of precipitation, as well as a larger gradient of $Z_{\mathrm{H}}$ above the ML. A thicker ML is also associated with a higher distance between the peak in $Z_{\mathrm{H}}$ and the minimum in $\rho_{\mathrm{hv}}$, a factor related to the concentration of hydrometeors. A higher concentration of particles will increase the diabatic cooling caused by the melting process, which could increase the thickness of the ML. Additionally, a significant dependency on the presence of rimed particles above the ML and the vertical velocity in the ML was found. Rimed particles are characterized by their higher density and their higher fall velocities, which leads to an increase in the distance travelled by a particle before complete melting.

Finally, a principal component analysis showed that the most important factors explaining the overall variability of the ML are (in order of importance) the fraction of riming, the gradient of $Z_{\mathrm{H}}$ in the solid phase, and values of $\rho_{\mathrm{hv}}$ inside the ML.

The analysis of the ML conducted during this work could be complemented with additional X-band radar datasets from other climatic regions, in order to verify the consistency of the characterization. Future work will be devoted to the extension of the algorithm to PPI and to other frequencies, in particular to the Swiss operational polarimetric C-band radar network.

\section{Acknowledgements}

This work is a contribution to the HyMeX programme. The authors thank Prof. W. F. Krajewski from the University of Iowa for providing some IFloodS radar data. The authors also thank MeteoSwiss Payerne, in particular B. Calpini, D. Ruffieux and G. Romanens for access to the Payerne radiosounding data. The authors are grateful to J. Grazioli for the processing of the raw radar data. Significant funding has been obtained from the Competence Center of Environment and Sustainibility (CCES) of the Eidgenössische Technische Hochschule (ETH) domain through the Open Support Platform for Environmental Research (OSPER) project.

\section{References}

Bandera J, Papatsoris AD, Watson PA, Tan J, Goddard JW. 1998. Method for detecting the extend of the melting layer. IET Electr. Lett. 11: 2104-2105, doi: 10.1049/el:19981462.

Bellon A, Zawadzki I, Fabry F. 1997. Measurements of melting layer attenuation at X-band frequencies. Radio Sci. 32: 943-955.

Brandes E, Ikeda K. 2004. Freezing-level estimation with polarimetric radar. J. Appl. Meteorol. 43: 1541-1553.

Chilès J-P, Delfiner P. 1999. Geostatistics: Modeling Spatial Uncertainty, Probability and Statistics. John Wiley \& Sons: Hoboken, NJ.

Das S, Maitra A, Shukla AK. 1993. Melting layer characteristics at different climatic conditions in the Indian region: Ground based measurements and satellite observations. Atmos. Res. 101: 78-83, doi: 10.1016/j.atmosres. 2011.01.013.

Domaszczynski P. 2012. 'Performance evaluation of a network of polarimetric $\mathrm{X}$-Band radars used for rainfall estimation', $\mathrm{PhD}$ thesis. University of Iowa: Iowa City, IA. 
Doviak R, Zrnić D. 2006. Doppler Radar and Weather Observations (2nd edn). Dover Publications: Mineola, NY.

Drobinski P, Ducrocq V, Alpert P, Anagnostou E, Béranger K, Borga M Braud I, Chanzy A, Davolio S, Delrieu G, Estournel C, Filali Boubrahm N, Font J, Grubisic V, Gualdi S, Homar V, Ivancan-Picek B, Kottmeier C, Kotroni V, Lagouvardos K, Lionello P, Llasat MC, Ludwig W, Lutoff C, Mariotti A, Richard E, Romero R, Rotunno R, Roussot O, Ruin I, Somot S, Taupier-Letage I, Tintore J, Uijlenhoet R, Wernli H. 2014. HyMeX, 10 -year multidisciplinary program on the Mediterranean water cycle. Bull. Am. Meteorol. Soc. 95: 1063-1082, doi: 10.1175/BAMS-D-12-00242.1.

Ducrocq V, Braud I, Davolio S, Ferretti R, Flamant C, Jansà A, Kalthoff N, Richard E, Taupier-Letage I, Ayral P-A, Belamari S, Berne A, Borga M, Boudevillain B, Bock O, Boichard J-L, Bouin M-N, Bousquet O, Bouvier C, Chiggiato J, Cimini D, Corsmeier U, Coppola L, Cocquerez P, Defer E, Delanoë J, Di Girolamo P, Doerenbecher A, Drobinski P, Dufournet Y, Fourrié N, Gourley JJ, Labatut L, Lambert D, Le Coz J, Marzano FS, Molinié G, Montani A, Nord G, Nuret M, Ramage K, Rison B, Roussot O, Said F, Schwarzenboeck A, Testor P, Van-Baelen J, Vincendon B, Aran M, Tamayo J. 2014. HyMeX-SOP1, the field campaign dedicated to heavy precipitation and flash flooding in the northwestern Mediterranean. Bull. Am. Meteorol. Soc. 95: 1083-1100, doi: 10.1175/BAMS-D-12-00244.1.

Durden SL, Kitiyakara A, Eastwood I, Tanner AB, Haddad ZS, Li FK, Wilson WJ. 1997. ARMAR observations of the melting layer during TOGA COARE. IEEE Trans. Geosci. Remote Sens. 35: 1453-1456.

Fabry F, Zawadzki I. 1995. Long term observations of the melting layer of precipitation and their interpretation. J. Atmos. Sci. 52: 838-851.

Giangrande SE, Krause JM, Ryzhkov AV. 2008. Automatic designation of the melting layer with a polarimetric prototype of the WSR-88D radar. J. Appl. Meteorol. Clim. 47: 1354-1364, doi: 10.1175/2007JAMC1634.1.

Grazioli J, Schneebeli M, Berne A. 2014a. Accuracy of phase-based algorithms for the estimation of the specific differential phase shift using simulated polarimetric weather radar data. IEEE Geosci. Remote Sens. Lett. 11: 763-767, doi: 10.1109/LGRS.2013.2278620.

Grazioli J, Tuia D, Berne A. 2014b. Hydrometeor classification from polarimetric radar measurements: A clustering approach. Atmos. Meas. Tech. Discuss. 7: 8465-8519, doi: 10.5194/amtd-7-8465-2014.

Hubbert M, Rousseeuw PJ, Branden KV. 2005. ROBPCA: A new approach to robust principal component analysis. Technometrics 47: 64-79.

Inman HF, Bradley EL. 1989. The overlapping coefficient as a measure of agreement between probability distributions and point estimation of the overlap of two normal densities. Commun. Stat. Theory Methods 18 3851-3874, doi: 10.1080/03610928908830127.

Kain JS, Goss S, Baldwin E. 2000. The melting effect as a factor in precipitationtype forecasting. Weather and Forecasting 15: 700-714.

Kalogiros J, Anagnostou MN, Anagnostou E, Montopoli M, Picciotti E, Marzano FS. 2013. Correction of polarimetric radar reflectivit measurements and rainfall estimates for apparent vertical profile in stratiform rain. J. Appl. Meteorol. Clim. 52: 1170-1186, doi: 10.1175/JAMCD-12-0140.1.

Klaassen W. 1990. Attenuation and reflection of radio waves by a melting layer of precipitation. IEEE Trans. Antennas Propag. 137: 39-44.

Marshall JS, Hitschfeld W, Gunn KLS. 1955. Advances in radar weather. Adv. Geophys. 2: 1-56.

Matrosov SY. 2008. Assessment of radar signal attenuation caused by the melting hydrometeor layer. IEEE Trans. Geosci. Remote Sens. 46: 1039-1047, doi: 10.1109/TGRS.2008.915757.

Matrosov SY, Clark KA, Kingsmill DE. 2007. A polarimetric radar approach to identify rain, melting-layer, and snow regions for applying corrections to vertical profiles of reflectivity. J. Appl. Meteorol. Clim. 46: 154-166.

Peel MC, Finlayson BL, McMahon TA. 2007. Updated world map of the Köppen-Geiger climate classification. Hydrol. Earth Syst. Sci. 11: 1633-1644, doi: 10.5194/hess-11-1633-2007.

Pruppacher HR, Klett RL. 1997. Microphysics of Clouds and Precipitation (2nd edn), Atmospheric and Oceanographic Sciences Library 18. Kluwer Academic Press: Berlin and Heidelberg, Germany.

Pujol O, Mesnard F, Sauvageot H. 2012. Effects of melting layer in airborne meteorological X-Band radar observations. IEEE Trans. Geosci. Remote Sens. 50: $2318-2324$.

Ryzhkov AV, Giangrande SE, Melnikov VM, Schuur TJ. 2005. Calibration issues of dual-polarization radar measurements. J. Atmos. Oceanic Technol. 22: 1138-1155, doi: 10.1175/JTECH1772.1

Sánchez-Diezma R, Zawadzki I, Sempere-Torres D. 2000. Identification of the bright band through the analysis of volumetric radar data. J. Geophys. Res. 105: 2225-2236, doi: 10.1029/1999JD900310.

Schleiss M, Jaffrain J, Berne A. 2012. Stochastic simulation of intermittent DSD fields in time. J. Hydrometeorol. 13: 621-637, doi: 10.1175/JHM-D11-018.1.

Schneebeli M, Grazioli J, Berne A. 2014. Improved estimation of the specific differential phase shift using a compilation of Kalman filter ensembles. IEEE Trans. Geosci. Remote Sens. 52: 5137-5149, doi: 10.1109/TGRS. 2013.2287017.

Testud J, Le Bouar E, Obligis E, Ali-Mehenni M. 2000. The rain profiling algorithm applied to polarimetric weather radar. J. Atmos. Oceanic Technol. 17: $332-356$.

Wenshuo G, Xiaoguang Z, Lei Y, Huizhong L. 2010. 'An improved Sobel edge detection'. In 3rd IEEE International Conference on Computer Science and Information Technology (ICCSIT), Chengdu, China. 5: 67-71, doi: 10.1109/ ICCSIT.2010.5563693.

White AB, Gottas DJ, Strem ET, Ralph FM, Neiman PJ. 2002. An automated brightband height detection algorithm for use with Doppler radar spectral moments. J. Atmos. Oceanic Technol. 19: 687-697.

Zawadzki I, Szyrmer W, Bell C, Fabry F. 2005. Modeling of the melting layer. Part III: The density effect. J. Atmos. Sci. 62: 3705-3723, doi: 10.1175/ JAS3563.1. 